\title{
Zero Cells of the Siegel-Gottschling Fundamental Domain of Degree 2
}

\author{
Takahiro Hayata, Takayuki Oda, and Tomoki Yatougo
}

\section{CONTENTS}

1. Introduction

2. Siegel's Fundamental Domain of Degree 2

3. Index-Oriented Model of the Boundary

4. Involutions

5. 0-Cells and Maximal Labels

6. Procedure to Obtain 0-Cells

7. Proof of Theorem 5.1

8. $\Gamma$-Equivalence Classes of Zero Cells

\section{References}

2000 AMS Subject Classification: Primary 11F46, Secondary 11F06 Keywords: Siegel modular group, Siegel upper half-space, fundamental domain, reduction theory
Let $\mathcal{F}_{n}$ be a fundamental domain of the Siegel upper half-space of degree $n$ with respect to the Siegel modular group $\operatorname{Sp}(n, \mathbb{Z})$. According to Siegel himself, $\mathcal{F}_{n}$ is determined by only finitely many polynomial inequalities. In case of degree $n=2$, Gottschling determined the minimal set of inequalities. The boundary of $\mathcal{F}_{2}$ is of great concern in the literature not only from a homological point of view but also from the geometry of numbers. In this paper we compute the vertices of $\mathcal{F}_{2}$ under the condition that the defining ideal is zero-dimensional ("0-cells"). We also discuss an equivalence relation among 0 -cells.

\section{INTRODUCTION}

In the classical study of elliptic modular forms, the fundamental domain

$$
\mathcal{F}_{1}=\left\{z=x+y \sqrt{-1} \in \mathbb{C}|| x\left|\leq \frac{1}{2}, y>0,\right| z \mid \geq 1\right\}
$$

is well known [Serre 73], and knowledge of the fundamental domain of an arithmetic subgroup plays an important role [Fricke and Klein 65]. But there seem to be few articles on the fundamental domains of classical symmetric domains of higher dimension. This paper is a case study for the next difficult case. Let $\mathcal{F}_{2}$ be the fundamental domain of the Siegel modular group $\operatorname{Sp}(2, \mathbb{Z})$ of genus 2 in the Siegel upper half-space $\mathbb{H}_{2}$. In his book [Siegel 64], C. L. Siegel proved that such a fundamental domain in general degree is determined by only finitely many inequalities of the form $|\operatorname{det}(C Z+D)| \geq 1$ and with the Minkowski condition [Klingen 90]. If the degree is 2, Gottschling determined the minimal set of inequalities [Gottschling 59]. In the following we specify the fundamental domain $\mathcal{F}_{2}$ determined by this minimal set as the Siegel-Gottschling fundamental domain.

In the literature, several papers are concerned with fixed points or conjugacy classes [Ueno 71, Ueno 72, Hashimoto 83]. Gottschling himself also computed fixed points and fixed-point subgroups of $\operatorname{Sp}(2, \mathbb{Z})$ [Gottschling 61b, Gottschling 61a]. In another direction, there is a paper [MacPherson and McConnell 93] on the 
topology of modular groups of genus 2 that shows the existence of a spine. See also [Yasaki 06]. There also is an attempt to develop a non-Euclidean Voronoi theory [Watanabe 03, Watanabe 11].

To continue the investigation of the fundamental domains, we are interested in the "fine structure" of this Siegel-Gottschling $\mathcal{F}_{2}$. The idea is as follows. We regard $\mathrm{H}_{2}$ as a real affine space $V_{\mathbb{R}}$ of dimension 6 . By the result of Siegel and Gottschling mentioned above, the boundary $\partial \mathcal{F}_{2}$ of the domain $\mathcal{F}_{2}$ consists of real 5 -dimensional hypersurfaces ("walls"), and each of them has a description by polynomial equations. To understand the cells that form $\partial \mathcal{F}_{2}$ is to understand the real zeros of the system of polynomial equations. Generally speaking, however, the detailed structure of the intersections of these walls seem to be unknown. Since $\operatorname{dim}_{\mathbb{R}} \mathcal{F}_{2}=6$, it seems to be very hard to obtain a complete answer to this problem.

In this paper we restrict ourselves to a consideration of the "0-cells" under the condition that the ideal defined by the system of walls is 0-dimensional. Our goal is to prove several results on these 0 -cells. To present the main result, we require some notation. By [Gottschling 59, Sätze 1 and 2], there are 28 walls defined by polynomial equations $f_{1}=0, \ldots, f_{28}=0$. For a label $L=\left\{i_{1}, \ldots, i_{p}\right\}$ $\left(1 \leq i_{j} \leq 28\right)$, let $I_{L}=\left\langle f_{i_{1}}, \ldots, f_{i_{p}}\right\rangle$ be the ideal in the polynomial ring $\mathbb{Q}[V]$ over the rational numbers $\mathbb{Q}$ of six variables. Let $V\left(I_{L}\right)$ be the zero set of $I_{L}$. Then we have the following theorem.

Theorem 1.1. There are 180 points $p \in V\left(I_{L}\right) \cap \partial \mathcal{F}_{2}$ for some $L$ such that $I_{L}$ is zero-dimensional. They are divided into $\operatorname{Sp}(2, \mathbb{Z})$-equivalence classes, and the upper bound on the number of classes is 40, the lower bound is 25 .

The points obtained as 0-cells seem to have a greater chance to be informative in view of Voronoi theory [Martinet 03] and its extensions. For example, the point $Z_{8}=\left(\begin{array}{cc}\eta & (\eta-1) / 2 \\ (\eta-1) / 2 & \eta\end{array}\right)$ with $\eta=(1+2 \sqrt{2} i) / 3$ in $\partial \mathcal{F}_{2}$ appears as a 0 -cell in $\partial \mathcal{F}_{2}$, which also appeared in Gottschling's paper. Our index model (Section 3) describes this 0-cell as lying on the intersection of three "rank-1" equalities, four "rank-2" equalities, and two Minkowski conditions, and is strictly positive in the other 19 inequalities. There is a notion of Hermite constant in the case of the linear algebraic group [Watanabe 03]. We can prove that $Z_{8}$ attains the minimum $\operatorname{det}(Y)$ among 180 points. We remark that it was announced in [Kawamura 09] that $Z_{8}$ attains the minimum $\operatorname{det}(Y)$ on
$\mathcal{F}_{2}$, which implies that $Z_{8}$ attains the Hermite constant of the symplectic group of degree 2 .

Now we explain the contents of the paper. In Section 2, we review Gottschling's theorem and fix notation. We introduce an index model based on the numbering of the walls in Section 3. We also introduce an involution that works efficiently during the classification process (Section 4). Then we state the main theorem of the paper in Section 5 . Since the results were obtained through exhaustive computer search, we explain the procedure using a computer algebra system step by step and give search results in Section 6 before the proof of the main theorem (Section 7). Lastly, in Section 8, we discuss the $\Gamma$-equivalence and inequivalence property of the set of 0-cells in detail.

The authors thank the referee for a very careful reading of our manuscript and many suggestions for corrections.

\section{SIEGEL'S FUNDAMENTAL DOMAIN OF DEGREE 2}

Let $\mathrm{H}_{2}$ be the Siegel upper half-space of degree 2, namely,

$$
\begin{array}{r}
\mathrm{H}_{2}=\left\{Z=X+\sqrt{-1} Y \in M_{2}(\mathbb{C}) \mid\right. \\
\left.{ }^{t} Z=Z, Y: \text { positive definite }\right\},
\end{array}
$$

where $M_{2}(\mathbb{C})$ is the set of $2 \times 2$ complex matrices. The discrete group $\Gamma=\operatorname{Sp}(2, \mathbb{Z})$ is a set of symplectic matrices of degree 4 whose entries are integers:

$$
\Gamma=\left\{g \in M_{4}(\mathbb{Z}) \mid{ }^{t} g\left(\begin{array}{cc}
0_{2} & 1_{2} \\
-1_{2} & 0_{2}
\end{array}\right) g=\left(\begin{array}{cc}
0_{2} & 1_{2} \\
-1_{2} & 0_{2}
\end{array}\right)\right\} .
$$

The matrix $\gamma \in \Gamma$ acts on $\mathrm{H}_{2}$ discontinuously by the linear fractional transformation

$$
\gamma \cdot Z=(A Z+B)(C Z+D)^{-1}, \quad \gamma=\left(\begin{array}{ll}
A & B \\
C & D
\end{array}\right) .
$$

Siegel's fundamental domain $\mathcal{F}_{2}$ of $\mathrm{H}_{2}$ with respect to the action of $\Gamma$ is given as follows [Klingen 90, Siegel 64]. Let $X_{i j}, Y_{i j}$ be the $(i, j)$-entries of the $2 \times 2$ matrices $X$, $Y$ respectively. By definition, $\mathcal{F}_{2}$ consists of the points $Z=X+\sqrt{-1} Y \in M_{2}(\mathbb{C})$ determined by the following three types of inequalities:

(1) $\left|X_{i j}\right| \leq 1 / 2$.

(2) $Y$ is Minkowski reduced [Klingen 90, Section I.2]). Specifically, $0 \leq 2 Y_{12} \leq Y_{11} \leq Y_{22}$.

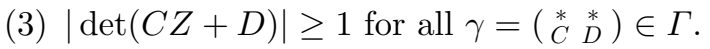

Siegel proved that it is enough to consider only a finite number of $\gamma^{\prime}$ s in the condition (3). Gottschling 
[Gottschling 59] determined a set of 15 inequalities out of (3) that form a minimal set.

To recall Gottschling's description, we prepare a set of square matrices of degree 2. Let $E_{i j}$ be the identity matrix and $O$ the zero matrix. For simplicity, we set

$$
\begin{aligned}
E_{1} & =E_{11}, \quad E_{2}=E_{22}, \quad I_{ \pm}=E_{1} \pm E_{2}, \\
J & =E_{12}+E_{21}, \quad J_{i}=J+E_{i} \quad(i=1,2) .
\end{aligned}
$$

Theorem 2.1. [Gottschling 59, Sätze 1, 2] The condition (3) is exhausted by the following matrix pairs $(C, D)$, and no proper subset of them is sufficient to define the fundamental domain $\mathcal{F}_{2}$ :

$$
\begin{aligned}
& \operatorname{rank}(C)=1: \\
& \begin{aligned}
(C, D) \in\{ & \left(E_{1}, E_{2}\right),\left(E_{2}, E_{1}\right),\left(E_{1}-E_{12}, I_{+}+E_{21}\right), \\
& \left.\left(E_{1}-E_{12},-I_{+}-E_{21}\right)\right\} ;
\end{aligned}
\end{aligned}
$$

$\operatorname{rank}(C)=2$ :

$$
(C, D) \text { with; } C=I_{+}, \quad \pm D=O, E_{1}, E_{2}, I_{ \pm}, J, J_{1}, J_{2} .
$$

\section{INDEX-ORIENTED MODEL OF THE BOUNDARY}

Let $\partial \mathcal{F}_{2}$ be the boundary of $\mathcal{F}_{2}$. We want to understand $\partial \mathcal{F}_{2}$ from the viewpoint of real algebraic geometry as semialgebraic subsets in $\mathcal{F}_{2}$. From the definition of $\mathcal{F}_{2}$, we introduce the defining polynomials $f_{\lambda}$ to describe $\partial \mathcal{F}_{2}$.

Now we define a finite set $\Lambda$ indexing the conditions (1), (2), and (3). For the condition (1), we set

$$
\begin{aligned}
f_{X_{1}}(Z) & =1 / 2-X_{11}, \quad f_{X_{2}}(Z)=1 / 2-X_{22}, \\
f_{X_{3}}(Z) & =1 / 2-X_{12} \quad f_{\theta X_{1}}(Z)=1 / 2+X_{11}, \\
f_{\theta X_{2}}(Z) & =1 / 2+X_{22}, \quad f_{\theta X_{3}}(Z)=1 / 2+X_{12} .
\end{aligned}
$$

Secondly, for the condition (2), we set

$$
f_{Y_{1}}(Z)=Y_{22}-Y_{11}, \quad f_{Y_{2}}(Z)=Y_{11}-2 Y_{12}, \quad f_{Y_{3}}(Z)=Y_{12} .
$$

Thirdly, we consider the condition (3) with the help of Theorem 2.1. For simplicity we set $\mathbf{1}=\left(E_{1}, E_{2}\right) \in \Lambda, \mathbf{2}=$ $\left(E_{2}, E_{1}\right) \in \Lambda, R=\left(E_{1}-E_{12}, I_{+}+E_{21}\right) \in \Lambda$, and $\theta R=$ $\left(E_{1}-E_{12},-I_{+}-E_{21}\right) \in \Lambda$ in the case of rank 1 in Theorem 2.1. For these $\lambda \in \Lambda$, we put $f_{\lambda}(Z)=\mid \operatorname{det}(C Z+$ $D)\left.\right|^{2}-1$. In the case of rank $2, C$ is always the identity. So we represent $(C, D) \in \Lambda$ by $D$. Then for $D \in \Lambda$, we put

$$
f_{D}(Z)=|\operatorname{det}(Z+D)|^{2}-1 .
$$

Thus $\Lambda$ consists of 28 elements: $6+3+4+15=28$.

Put $W_{\lambda}=\left\{Z \in \mathcal{F}_{2} \mid f_{\lambda}(Z)=0\right\}$. Obviously, $\partial \mathcal{F}_{2}=$ $\cup_{\lambda \in \Lambda} W_{\lambda}$. Moreover, define the extended notation $W_{L}=$ $\bigcap_{\lambda \in L} W_{\lambda}$ for a subset $L$ in $\Lambda$. Note that the labels are inclusion-reversing, i.e., $L \subset L^{\prime} \Rightarrow W_{L} \supset W_{L^{\prime}}$.

Though we do not know the substance of the labeled subset $W_{L}$ of $\partial \mathcal{F}_{2}$, we hope that the contiguity of $W_{L}$ are useful to parameterize the cells in $\partial F_{2}$. Besides, we can see the substance of $W_{L}$ pretty much in the 0-dimensional case. We remark that the naive expectation $\operatorname{dim} W_{L}=$ $6-|L|$ is not true for $|L| \geq 2$.

Let us introduce a notion of 0-cells, a candidate for "vertices" in this model. Let $V$ be the affine space in which the coordinate $\left(X_{11}, X_{12}, X_{22}, Y_{11}, Y_{12}, Y_{22}\right)$ lives. Put $I_{L}=\left\langle f_{\lambda} \mid \lambda \in L\right\rangle, L \subset \Lambda$, for the ideal generated by $L$ and consider the zero set $V\left(I_{L}\right)$. Then one has $W_{L}=$ $V\left(I_{L}\right) \cap \mathcal{F}_{2}$.

\section{Definition 3.1.}

1. A label $L$ or $W_{L}$ is called a 0 -cell if the dimension of $V\left(I_{L}\right)$ is zero.

2. A label $L$ is called trivial if the corresponding ideal $I_{L}$ is trivial (i.e., $I_{L} \ni 1$ ).

\section{INVOLUTIONS}

We set $F_{\infty}(z)=-\bar{z}$ for $z \in \mathbb{C}$. We introduce involutive real-analytic diffeomorphisms $\theta_{i}, i=1,2,3$, on $\uplus_{2}$ by

$$
\begin{aligned}
\theta_{1}(Z) & =\left(\begin{array}{cc}
F_{\infty}\left(Z_{11}\right) & Z_{12} \\
Z_{12} & Z_{22}
\end{array}\right), \\
\theta_{2}(Z) & =\left(\begin{array}{cc}
Z_{11} & Z_{12} \\
Z_{12} & F_{\infty}\left(Z_{22}\right)
\end{array}\right), \\
\theta_{3}(Z) & =\left(\begin{array}{cc}
Z_{11} & F_{\infty}\left(Z_{12}\right) \\
F_{\infty}\left(Z_{12}\right) & Z_{22}
\end{array}\right) .
\end{aligned}
$$

Moreover we put $\theta(Z)=\theta_{1} \theta_{2} \theta_{3}(Z)$, which is a normalizer of $\Gamma$ in the group of diffeomorphisms of $\mathrm{H}_{2}$. For $\gamma=$ $\left(\begin{array}{ll}J & 0 \\ 0 & J\end{array}\right)$, we also define $\sigma_{0}(Z)=\gamma \cdot Z=\left(\begin{array}{ll}Z_{22} & Z_{12} \\ Z_{12} & Z_{11}\end{array}\right)$. Let $\Delta$ be the finite group of order 16 generated by $\theta_{i}, i=1,2,3$, and $\sigma_{0}$ with relation $\sigma_{0} \theta_{1} \sigma_{0}=\theta_{2}$. We say that two points $Z, Z^{\prime}$ in $\mathrm{H}_{2}$ are $\Delta$-equivalent if there is a $\delta \in \Delta$ such that $\delta(Z)=Z^{\prime}$, and this fact is denoted by $Z \stackrel{\Delta}{\sim} Z^{\prime}$. In each $\Delta$-equivalence class, we may choose a representative point $Z$ whose $X_{i j}$-coordinates are all nonnegative and $X_{11} \geq X_{22}$.

We define $f_{\theta \lambda}(Z)=f_{\lambda}(\theta Z)$. Then we have a more economical description of $\Lambda$ :

$$
\begin{aligned}
\Lambda=\left\{\mathbf{1}, \mathbf{2}, Y_{1}, Y_{2}, Y_{3}, O\right\} \cup\{D, \theta D \mid \\
\left.\quad D=R, I_{+}, I_{-}, J, E_{i}, J_{i}(i=1,2), X_{k}(k=1,2,3)\right\} .
\end{aligned}
$$




\section{0-CELLS AND MAXIMAL LABELS}

In this section we exhaust the whole set of 0-cells in our definition. The procedure consists of a few steps; each step is carried out by a computer algebra system. We enumerate all the possible $L$ such that $\operatorname{dim}\left(V_{L}\right)=0$ to get a finite set $V_{0}=\cup_{\operatorname{dim} V\left(I_{L}\right)=0} V\left(I_{L}\right)_{\mathbb{R}}$. A detailed explanation of the method and the computer computations are presented in Section 6. There are 752370 possibilities by the results of step 1 .

Next, for each $L$ such that $\operatorname{dim} V\left(I_{L}\right)=0$, we check $W_{L}=V\left(I_{L}\right)_{\mathbb{R}} \cap \partial \mathcal{F}_{2} \neq \varnothing$ in the finite set $V_{0}$. We explain the details of the method by an example in Section 7 . The corresponding algorithm is given in Section 6 (step 2).

Then the number of remaining $L$ such that $W_{L}$ is nonempty is 2146 , and we find that the set

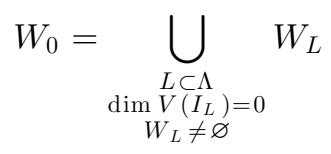

consists of 180 points. Lastly, we check the maximality condition (3) for $L$ in Theorem 5.1 below.

To state the main result, we define 180 points and their associated labels a priori, which are to be 0-cells. Define $e_{i}, 0 \leq i \leq 39$, as given in Table 1 .

In the table, we put $\tau(x)=\sqrt{1-x^{2}}$ and set $\omega_{j}$ to be the algebraic number given by a real root of $\Omega_{j}$ specified uniquely by indicated additional conditions in Tables 2 and 3. Their floating-point expressions are given in Table 4. Finally, we define 180 actual points using the action of $\Delta$. With a given point $p$, we associate a label $L_{p}$. They both are given in Tables 5 and 6 . The points $e_{i}$ are the representatives of $40 \Delta$-equivalence classes.

Summing up, we can state the main result of this paper.

Theorem 5.1. Let $p$ be a point and $L=L_{p}$ the label associated with $p$ in Tables 5 and 6. Then we have

(1) $I_{L}$ is zero-dimensional.

(2) $p \in W_{L}$.

(3) $L$ is maximal, i.e., $p \notin W_{L^{\prime}}$ for $L^{\prime} \supsetneq L$.

Computation of $\operatorname{det}(Y)$ from $p=X+\sqrt{-1} Y$ gives the following corollary.

Corollary 5.2. The points $\theta_{3} e_{1}$ and $\theta \theta_{3} e_{1}$ attain the minimum $\operatorname{det}(Y)$ among the 180 points in Tables 5 and 6.
Remark 5.3. Theorem 5.1 does not necessarily mean that we have a set of $L$ 's such that $\operatorname{dim}\left(W_{L}\right)=0$. Specifically, there could be $L \subset \Lambda$ such that $\operatorname{dim} W_{L}=0$ but $\operatorname{dim} V\left(I_{L}\right)>0$.

\section{PROCEDURE TO OBTAIN O-CELLS}

\subsection{The Number of Nontrivial Labels}

Logically speaking, the computation in this subsection is not necessary, but it is helpful in grasping the complexity of the computations.

Define $T^{(n)}$ to be the set of trivial labels with size $n$ (cf. Definition 3.1). We put $T_{\text {new }}^{(n)}=T^{(n)} \backslash \bigcup_{k<n} T^{(k)}$. Obviously, $T^{(1)}=\varnothing$. It is also clear the trivial labels of size 2 are

$$
T_{\text {new }}^{(2)}=T^{(2)}=\left\{\left[X_{1}, \theta X_{1}\right],\left[X_{3}, \theta X_{3}\right],\left[X_{2}, \theta X_{2}\right]\right\} .
$$

By computer search, we obtain $\left|T_{\text {new }}^{(3)}\right|=0,\left|T_{\text {new }}^{(4)}\right|=16$, $\left|T_{\text {new }}^{(5)}\right|=64,\left|T_{\text {new }}^{(6)}\right|=1024$.

The nontrivial labels are obtained as

$$
2^{\Lambda} \backslash \bigcup_{n \geq 2} \bigcup_{L \in T^{(n)}}\left\{L^{\prime} \in 2^{\Lambda} \mid L \subset L^{\prime}\right\}
$$

The inclusion-exclusion principle computes the cardinality. We restrict ourselves to the case $n \leq 4$. If we put $T=T^{(2)} \cup T_{\text {new }}^{(4)}$, then the cardinality of possibly nontrivial labels is given by $2^{28}-204166144=64269312$.

To clarify the situation of nontrivial labels of smaller size, we employ the inclusion-exclusion principle again. If the size of the labels is 2 , because $\left|T^{(2)}\right|=3$, the number of remaining labels of size 2 is $\left(\begin{array}{c}28 \\ 2\end{array}\right)-3=375$. If three, the number is $\left(\begin{array}{c}28 \\ 3\end{array}\right)-3\left(\begin{array}{c}26 \\ 1\end{array}\right)=3198$. If four, because $\left|T_{\text {new }}^{(4)}\right|=16$, therefore $\left(\begin{array}{c}28 \\ 4\end{array}\right)-3\left(\begin{array}{c}26 \\ 2\end{array}\right)-16+3=19487$. If five and six, we have respectively $\left(\begin{array}{c}28 \\ 5\end{array}\right)-8248+97-7=$ 90122 and $\left(\begin{array}{c}28 \\ 6\end{array}\right)-51762+1699-197+2=326482$ for the numbers of nontrivial labels. In conclusion, we have the following result:

\begin{tabular}{cccccc}
$|L|$ & 2 & 3 & 4 & 5 & 6 \\
\hline$\left(\begin{array}{c}28 \\
|L|\end{array}\right)$ & 378 & 3276 & 20475 & 98280 & 376740 \\
\# of nontrivial labels & 375 & 3198 & 19487 & 90122 & 326482
\end{tabular}

\subsection{The 0 -Dimensional Ideals}

Here, we collect the steps to obtain the 0-cells.

Procedure 6.1. Consider the nontrivial labels $L$.

step 1. Collect the $L$ 's where $I_{L}$ is zero-dimensional.

step 2. For each $L$ such that $I_{L}$ is zero-dimensional, compute all real zeros $\left\{p_{k, L}\right\}_{k} \in V\left(I_{L}\right)$. 


\begin{tabular}{|c|c|c|c|c|c|c|}
\hline$j$ & $X_{11}$ & $X_{12}$ & $X_{22}$ & $Y_{11}$ & $Y_{12}$ & $Y_{22}$ \\
\hline $\begin{array}{l}e_{0} \\
\end{array}$ & $1 / 2$ & 0 & $1 / 2$ & $\omega_{0}$ & 0 & $\omega_{0}$ \\
\hline$e_{1}$ & $1 / 3$ & $1 / 3$ & $1 / 3$ & $\omega_{1}$ & $\omega_{1} / 2$ & $\omega_{1}$ \\
\hline$e_{2}$ & $\omega_{6}$ & $1 / 2$ & $\omega_{6}$ & 1 & $1 / 2$ & 1 \\
\hline$e_{3}$ & $1 / 2$ & $1 / 2$ & $1 / 2$ & $\omega_{0}$ & 0 & $\omega_{0}$ \\
\hline$e_{4}$ & $1 / 2$ & $1 / 2$ & 0 & 1 & $1 / 2$ & 1 \\
\hline$e_{5}$ & $1 / 2$ & 0 & $1 / 2$ & 1 & $1 / 2$ & 1 \\
\hline$e_{6}$ & 0 & $1 / 2$ & 0 & 1 & $1 / 2$ & 1 \\
\hline$e_{7}$ & $1 / 2$ & $\omega_{0}-3 / 4$ & $1 / 2$ & $\omega_{0}$ & $\omega_{0} / 2$ & $\left(3-\omega_{0}\right) / 2$ \\
\hline$e_{8}$ & $1 / 2$ & $1 / 2$ & $1 / 2$ & $\omega_{3}$ & $\omega_{3} / 2$ & $\omega_{3}$ \\
\hline$e_{9}$ & $1 / 2$ & $1 / 2$ & $1 / 2$ & $\omega_{0}$ & $\omega_{0} / 2$ & $\omega_{4}$ \\
\hline$e_{10}$ & $1 / 2$ & $1 / 2$ & 0 & $\omega_{0}$ & $\omega_{0} / 2$ & $\omega_{5} / 16$ \\
\hline$e_{11}$ & $1 / 2$ & 0 & $1 / 2$ & $\omega_{0}$ & $\omega_{0} / 2$ & $\omega_{5} / 16$ \\
\hline$e_{12}$ & $\omega_{2}$ & $\left(1-\omega_{2}\right) / 2$ & $\omega_{2}$ & $\tau\left(\omega_{2}\right)$ & $\tau\left(\omega_{2}\right) / 2$ & $\tau\left(\omega_{2}\right)$ \\
\hline$e_{13}$ & $\left(1-3 \omega_{2}\right) / 2$ & $\left(1-\omega_{2}\right) / 2$ & $\omega_{2}$ & $\tau\left(\omega_{2}\right)$ & $\tau\left(\omega_{2}\right) / 2$ & $\tau\left(\omega_{2}\right)$ \\
\hline$e_{14}$ & $\left(1-3 \omega_{2}\right) / 2$ & $\omega_{2}$ & $\left(1-3 \omega_{2}\right) / 2$ & $\tau\left(\omega_{2}\right)$ & $\tau\left(\omega_{2}\right) / 2$ & $\tau\left(\omega_{2}\right)$ \\
\hline$e_{15}$ & $\omega_{2}$ & $\left(1-3 \omega_{2}\right) / 2$ & $\omega_{2}$ & $\tau\left(\omega_{2}\right)$ & $\tau\left(\omega_{2}\right) / 2$ & $\tau\left(\omega_{2}\right)$ \\
\hline$e_{16}$ & $1 / 2$ & $\omega_{7} / 4$ & $\left(\omega_{7}+1\right) / 2-\omega_{8} / 8$ & $\omega_{0}$ & $\omega_{0} / 2$ & $\frac{\omega_{0}\left(\omega_{8}+4-2 \omega_{7}\right)}{4}$ \\
\hline$e_{17}$ & $1 / 2$ & $\left(2-\omega_{7}\right) / 4$ & $\omega_{8} / 8$ & $\omega_{0}$ & $\omega_{0} / 2$ & $\frac{\omega_{0}\left(\omega_{8}+4-2 \omega_{7}\right)}{4}$ \\
\hline$e_{18}$ & $1 / 2$ & $1 / 2$ & $1 / 2$ & $\omega_{0}$ & $\omega_{21}$ & $\omega_{0}$ \\
\hline$e_{19}$ & $1 / 2$ & $1 / 4+\omega_{0} \omega_{21}$ & $\left(\omega_{0}-\omega_{21}\right)^{2} / 2$ & $\omega_{0}$ & $\left(\omega_{0}-\omega_{21}\right) / 2$ & $\tau\left(\left(\omega_{0}-\omega_{21}\right)^{2} / 2\right)$ \\
\hline$e_{20}$ & $\left(\omega_{0}-\omega_{21}\right)^{2} / 2$ & $\left(\omega_{0}-\omega_{21}\right)^{2} / 2$ & $\left(\omega_{0}-\omega_{21}\right)^{2} / 2$ & $\tau\left(\left(\omega_{0}-\omega_{21}\right)^{2} / 2\right)$ & $\frac{\omega_{0}}{2}+\frac{\omega_{21}}{4}-\omega_{21}^{3}$ & $\tau\left(\left(\omega_{0}-\omega_{21}\right)^{2} / 2\right)$ \\
\hline$e_{21}$ & $2 \omega_{14}$ & $1 / 2$ & $\omega_{14}$ & $\tau\left(\omega_{14}\right)$ & $\tau\left(\omega_{14}\right) / 2$ & $\tau\left(\omega_{14}\right)$ \\
\hline$e_{22}$ & $\omega_{15}$ & $1 / 2$ & $\omega_{16}$ & $\tau\left(\omega_{15}\right)$ & $\tau\left(\omega_{15}\right) / 2$ & $\tau\left(\omega_{15}-\omega_{16}\right)$ \\
\hline$e_{23}$ & $\omega_{17}$ & $1 / 2$ & $\omega_{17}$ & $\tau\left(\omega_{17}\right)$ & $\tau\left(\omega_{17}\right) / 2$ & $\tau\left(\omega_{17}\right)$ \\
\hline$e_{24}$ & $\omega_{20}$ & $\left(1-\omega_{20}\right) / 2$ & $1 / 2$ & $\tau\left(\omega_{20}\right)$ & $\tau\left(\omega_{20}\right) / 2$ & $\tau\left(\omega_{20} / 2\right)$ \\
\hline$e_{25}$ & $1 / 2$ & $\left(1-\omega_{18}\right) / 4$ & $1 / 2$ & $\omega_{19}$ & $\omega_{19} / 2$ & $\omega_{19}$ \\
\hline$e_{26}$ & $1 / 2$ & $\left(1+\omega_{18}\right) / 4$ & $\left(1-\omega_{18}\right) / 2$ & $\omega_{19}$ & $\omega_{19} / 2$ & $\omega_{19}$ \\
\hline$e_{27}$ & $\omega_{22}$ & $\omega_{23} / 2$ & $1 / 2$ & $\tau\left(\omega_{22}\right)$ & $\tau\left(\omega_{22}\right) / 2$ & $\tau\left(\omega_{22}\right) \omega_{24} / 2$ \\
\hline$e_{28}$ & $\omega_{25}$ & $1 / 2$ & $\omega_{26}$ & $\tau\left(\omega_{25}\right)$ & $\tau\left(\omega_{25}\right) / 2$ & $\tau\left(\omega_{26}\right)$ \\
\hline$e_{29}$ & $1 / 2$ & $1 / 2$ & 0 & $\omega_{0}$ & $\omega_{27} / 2$ & 1 \\
\hline$e_{30}$ & $\frac{1-\omega_{27}}{2}$ & $1 / 2$ & $\frac{1-\omega_{27}}{2}$ & $\tau\left(\frac{1-\omega_{27}}{2}\right)$ & $\tau\left(\frac{1-\omega_{27}}{2}\right)-\frac{1}{2}$ & $\tau\left(\frac{1-\omega_{27}}{2}\right)$ \\
\hline$e_{31}$ & $\omega_{28}$ & $1 / 2$ & $\omega_{28} / 2$ & $\tau\left(\omega_{28}\right)$ & $\tau\left(\omega_{28}\right) / 2$ & $\tau\left(\omega_{28} / 2\right)$ \\
\hline$e_{32}$ & $\omega_{29}$ & $\omega_{30}$ & $\omega_{29}$ & $\tau\left(\omega_{29}\right)$ & $\tau\left(\omega_{29}\right) / 2$ & $\tau\left(\omega_{29}\right)$ \\
\hline$e_{33}$ & $1-2 \omega_{30}$ & $1-\omega_{29}-\omega_{30}$ & $\omega_{29}$ & $\tau\left(\omega_{29}\right)$ & $\tau\left(\omega_{29}\right) / 2$ & $\tau\left(\omega_{29}\right)$ \\
\hline$e_{34}$ & $1-2 \omega_{30}$ & $\omega_{30}-\omega_{29}$ & $\omega_{29}$ & $\tau\left(\omega_{29}\right)$ & $\tau\left(\omega_{29}\right) / 2$ & $\tau\left(\omega_{29}\right)$ \\
\hline$e_{35}$ & $\omega_{31}$ & $\left(1+\omega_{31}-\omega_{32}\right) / 2$ & $\omega_{32} / 2$ & $\tau\left(\omega_{31}\right)$ & $\tau\left(\omega_{31}\right) / 2$ & $\tau\left(\omega_{32} / 2\right)$ \\
\hline$e_{36}$ & $\omega_{31}$ & $\left(1-\omega_{31}-\omega_{32}\right) / 2$ & $\omega_{32} / 2$ & $\tau\left(\omega_{31}\right)$ & $\tau\left(\omega_{31}\right) / 2$ & $\tau\left(\omega_{32} / 2\right)$ \\
\hline$e_{37}$ & $\omega_{33}$ & $\omega_{34} / 2$ & $\omega_{35} / 4$ & $\tau\left(\omega_{33}\right)$ & $\tau\left(\omega_{33}\right) / 2$ & $\tau\left(\omega_{35} / 4\right)$ \\
\hline$e_{38}$ & $\omega_{33}$ & $\omega_{34} / 2-\omega_{33}$ & $\omega_{33}-\omega_{34}-\frac{\omega_{35}}{4}+1$ & $\tau\left(\omega_{33}\right)$ & $\tau\left(\omega_{33}\right) / 2$ & $\tau\left(\omega_{35} / 4\right)$ \\
\hline$e_{39}$ & $1 / 2$ & $\omega_{38} / 2$ & $\omega_{36} / 2$ & $\tau\left(\omega_{36} / 2\right)$ & $\sqrt{\omega_{37}} / 2$ & $\tau\left(\omega_{36} / 2\right)$ \\
\hline
\end{tabular}

TABLE 1. Definition of the points $e_{j}=X+\sqrt{-1} Y(0 \leq j \leq 39)$.

step 3. For each point $p_{k, L}$, take the maximal $L \subset L_{\max }$ by inclusion. Register $\left(p_{k, L}, L_{\max }\right)$.

step 4. For each $(p, L)$ registered, if $p \in W_{L}$, then output $(p, L)$.

The ideal $I$ is 0 -dimensional if and only if $\operatorname{dim} \mathbb{Q}[V] / I<\infty$. So by computing the Gröbner basis of $I$ and checking its leading exponents, one can decide on the zero-dimensionality. Using the computer algebra system ASIR,${ }^{1}$ this is achieved by the command zero_dim in

${ }^{1}$ Available at http://www.math.kobe-u.ac.jp/asir/asir.html. the package gr. By an exhaustive search in $2^{\Lambda}$, we obtain the following search result.

Search Result 6.2. There are 752370 labels $L$ out of $2^{\Lambda}$, so that $I_{L}$ is zero-dimensional.

\subsection{The Minimal Polynomials of Ideals}

For each $L$ such that $I_{L}$ is zero-dimensional, we want to compute all real zeros $\left\{p_{k}\right\} \in V\left(I_{L}\right)$.

In preparation for step 2, we compute the minimal polynomial of the ideal $I$, which we now review. The propositions below are taken from 


$$
\begin{aligned}
& \Omega_{0}(t)=4 t^{2}-3, \quad \omega_{0}=\sqrt{3} / 2=0.866 \ldots \\
& \Omega_{1}(t)=9 t^{2}-8, \quad \omega_{1}=2 \sqrt{2} / 3=0.9428 \ldots \\
& \Omega_{2}(t)=8 t^{3}-13 t^{2}+10 t-1, \quad \omega_{2}=0.1163 \ldots \\
& \Omega_{3}(t)=9 t^{4}+4 t^{2}-16, \quad \omega_{3}=\frac{1}{3} \sqrt{2 \sqrt{37}-2}=1.0627 \ldots \\
& \Omega_{4}(t)=65536 t^{4}-133376 t^{2}+61009, \quad \omega_{4}=\sqrt{(3 \sqrt{3045}+521) / 2^{9}}=1.1579 \ldots \\
& \Omega_{5}(t)=t^{4}-441 t^{2}+42849, \quad \omega_{5}=3 \sqrt{(\sqrt{285}+49) / 2}=17.2 \ldots \\
& \Omega_{6}(t)=4 t^{4}-8 t^{3}+12 t^{2}-8 t+1, \quad \omega_{6}=0.1593 \ldots \\
& \Omega_{7}(t)=t^{4}+22 t^{2}+96 t-39, \quad \omega_{7}=0.3739 \ldots \\
& \Omega_{8}(t)=t^{4}-14 t^{3}+11 t^{2}+2250 t-3687, \quad \omega_{8}=\left(3+10 \omega_{7}-\omega_{7}^{2}\right) / 4=1.6500 \ldots \\
& \Omega_{14}(t)=9 t^{4}-24 t^{3}+68 t^{2}-48 t+4, \quad \omega_{14}=0.0959 \ldots \\
& \Omega_{15}(t)=9 t^{4}-24 t^{3}+52 t^{2}-32 t+4, \quad \omega_{15}=0.1670 \ldots \\
& \Omega_{16}(t)=1296 t^{4}-3744 t^{3}+5224 t^{2}-4504 t+505, \quad \omega_{16}=\left(3 \omega_{15}^{3}-6 \omega_{15}^{2}+16 \omega_{15}-2\right) / 4=0.1299 \ldots \\
& \Omega_{17}(t)=15 t^{4}-32 t^{3}-4 t^{2}+32 t-4, \quad \omega_{17}=0.1291 \ldots \\
& \Omega_{18}(t)=t^{6}-2 t^{4}+8 t^{3}-259 t^{2}+256 t-64, \quad \omega_{18}=0.5285 \ldots \\
& \Omega_{19}(t)=2304 t^{12}+3712 t^{10}-1776 t^{8}-2848 t^{6}-1016 t^{4}-240 t^{2}+225, \quad \omega_{19}=\left(1-\omega_{18}\right) /\left(2 \sqrt{2 \omega_{18}-1}\right)=0.9861 \ldots \\
& \Omega_{20}(t)=4 t^{5}+7 t^{4}-28 t^{3}-38 t^{2}+48 t-9, \quad \omega_{20}=0.241164 \ldots \\
& \Omega_{21}(t)=256 t^{8}-256 t^{6}+224 t^{4}-848 t^{2}+25, \quad \omega_{21}=0.1723559 \ldots \\
& \Omega_{22}(t)=9 t^{6}-2 t^{5}-57 t^{4}+107 t^{2}+2 t-23, \quad \omega_{22}=0.4843 \ldots \\
& \Omega_{23}(t)=9 t^{6}+44 t^{5}-36 t^{4}-52 t^{3}+124 t^{2}+24 t-12, \quad \omega_{23}=\sqrt{2+2 \omega_{22}}-1-\omega_{22}=0.2386 \ldots \\
& \Omega_{24}(t)=12 t^{6}-88 t^{5}+148 t^{4}+28 t^{3}-120 t^{2}+36 t+23, \quad \omega_{24}=\omega_{22}^{5}+\left(37 \omega_{22}^{4}-148 \omega_{22}^{3}-152 \omega_{22}^{2}+130 \omega_{22}+73\right) / 36=2.402 \ldots \\
& \Omega_{25}(t)=7 t^{12}-16 t^{11}-44 t^{10}+368 t^{9}-1804 t^{8}+3840 t^{7}-3744 t^{6}-3200 t^{5} \\
& +12560 t^{4}-6912 t^{3}-6848 t^{2}+6912 t-1344, \quad \omega_{25}=0.2914 \ldots \\
& \Omega_{26}(t)=7168 t^{12}-34816 t^{11}+20224 t^{10}+231936 t^{9}-599760 t^{8}+222816 t^{7}+713128 t^{6}+31320 t^{5}-1793601 t^{4} \\
& +1157252 t^{3}+463714 t^{2}-470548 t+4511 \\
& \omega_{26}=\left(3997 \omega_{25}^{11}-4999 \omega_{25}^{10}-31374 \omega_{25}^{9}+180128 \omega_{25}^{8}-836696 \omega_{25}^{7}+1270256 \omega_{25}^{6}-551200 \omega_{25}^{5}-2995952 \omega_{25}^{4}\right. \\
& \left.+4650320 \omega_{25}^{3}+1295920 \omega_{25}^{2}-4073760 \omega_{25}+989888\right) / 572416=0.0096 \ldots \\
& \Omega_{27}(t)=t^{8}+4 t^{6}-16 t^{5}-42 t^{4}-32 t^{3}+164 t^{2}-16 t-47, \quad \omega_{27}=0.7113 \ldots \\
& \Omega_{28}(t)=9 t^{8}-24 t^{7}+56 t^{6}+16 t^{5}-232 t^{4}-416 t^{3}+1760 t^{2}-1088 t+144, \quad \omega_{28}=0.1851 \ldots \\
& \Omega_{29}(t)=t^{8}-4 t^{7}+16 t^{6}-20 t^{5}-40 t^{4}+64 t^{3}+28 t^{2}-40 t+4, \quad \omega_{29}=0.1105 \ldots \\
& \Omega_{30}(t)=256 t^{8}-512 t^{7}+1280 t^{6}-1536 t^{5}+1888 t^{4}-1120 t^{3}+512 t^{2}+96 t-87 \\
& \omega_{30}=\left(3 \omega_{29}^{7}-11 \omega_{29}^{6}+59 \omega_{29}^{5}-88 \omega_{29}^{4}+78 \omega_{29}^{3}-2 \omega_{29}^{2}-188 \omega_{29}+74\right) / 132=0.4036 \ldots \\
& \Omega_{31}(t)=t^{16}-12 t^{15}+88 t^{14}-448 t^{13}+1716 t^{12}-5104 t^{11}+11896 t^{10}-21956 t^{9}+32228 t^{8}-37776 t^{7}+35464 t^{6} \\
& -25968 t^{5}+14736 t^{4}-5744 t^{3}+1220 t^{2}-120 t+4, \quad \omega_{31}=0.18587 \ldots
\end{aligned}
$$

TABLE 2. Minimal polynomials of algebraic numbers appearing in the coordinates. (Note that we do not use the labels $\Omega_{i}$, $\omega_{i}$ $(9 \leq i \leq 13)$.)

[Noro and Yokoyama 03, Saito et al. 03] with terminology there and without proof. Let $P=\mathbb{Q}[V]=$ $\mathbb{Q}\left[X_{1}, \ldots, X_{n}\right]$ be a polynomial ring of $n$-variables. Let $I$ be an ideal of $P$. Let $f \in P$. We say that $m(I, f ; t) \in \mathbb{Q}[t]$ is a minimal polynomial of $I$ with respect to $f$ if

$$
\{g(t) \in P \mid g(f) \in I\}=\langle m(I, f ; t)\rangle .
$$

Proposition 6.3. If $I$ is zero-dimensional, then there is a unique $m(I, f ; t)$ with respect to every $f \in P$.
Proposition 6.4. If $f(X)=X_{i}$ is a monomial of degree 1 , then

$$
\left\{p \in V(\mathbb{C}) \mid m\left(I, X_{i} ; p_{i}\right)=0 \text { for all } i\right\} \supset V(I)
$$

This proposition implies that we have candidates for points of $V(I)$ by combining the zeros of the minimal polynomials of the indeterminates. As for determining the actual zeros $V(I)$, we have the following result. 


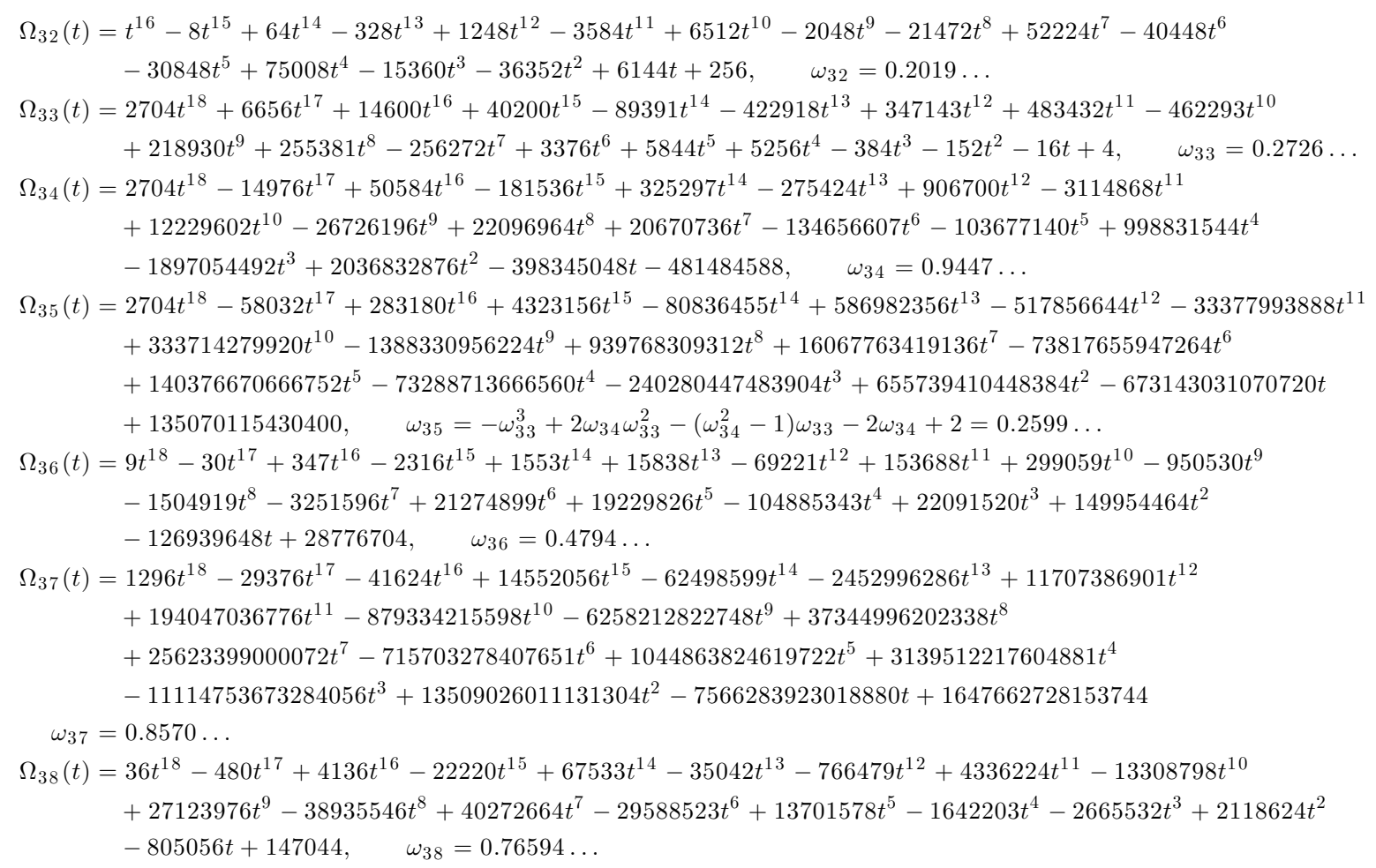

TABLE 3. Minimal polynomials of algebraic numbers (2).

\begin{tabular}{l|lr|l} 
pts & coordinate expression & pts & coordinate expression \\
\hline$e_{0}$ & {$[0.5,0,0.5,0.866,0,0.866]$} & $e_{20}$ & {$[0.240,0.240,0.240,0.9706,0.4709,0.9706]$} \\
$e_{1}$ & {$[0.333,0.333,0.333,0.942,0.471,0.942]$} & $e_{21}$ & {$[0.191,0.5,0.095,0.9953,0.4976,0.9953]$} \\
$e_{2}$ & {$[0.159,0.5,0.159,1,0.5,1]$} & $e_{22}$ & {$[0.167,0.5,0.1299,0.985,0.4929,0.9993]$} \\
$e_{3}$ & {$[0.5,0.5,0.5,0.866,0,0.866]$} & $e_{23}$ & {$[0.1291,0.5,0.1291,0.9916,0.495,0.9916]$} \\
$e_{4}$ & {$[0.5,0.5,0,1,0.5,1]$} & $e_{24}$ & {$[0.2411,0.3794,0.5,0.9704,0.4852,0.992]$} \\
$e_{5}$ & {$[0.5,0,0.5,1,0.5,1]$} & $e_{25}$ & {$[0.5,0.117,0.5,0.9861,0.4930,0.9861]$} \\
$e_{6}$ & {$[0,0.5,0,1,0.5,1]$} & $e_{26}$ & {$[0.5,0.3821,0.2357,0.9861,0.4930,0.9861]$} \\
$e_{7}$ & {$[0.5,0.1160,0.5,0.866,0.433,1.066]$} & $e_{27}$ & {$[0.4843,0.1193,0.5,0.8748,0.4374,1.0508]$} \\
$e_{8}$ & {$[0.5,0.5,0.5,1.062,0.531,1.062]$} & $e_{28}$ & {$[0.2914,0.5,0.0096,0.9565,0.4782,0.9999]$} \\
$e_{9}$ & {$[0.5,0.5,0.5,0.866,0.433,1.157]$} & $e_{29}$ & {$[0.5,0.5,0,0.866,0.3556,1]$} \\
$e_{10}$ & {$[0.5,0.5,0,0.866,0.433,1.076]$} & $e_{30}$ & {$[0.1443,0.5,0.1443,0.9895,0.4895,0.9895]$} \\
$e_{11}$ & {$[0.5,0,0.5,0.866,0.433,1.076]$} & $e_{31}$ & {$[0.1851,0.5,0.092,0.9827,0.4913,0.9957]$} \\
$e_{12}$ & {$[0.1163,0.441,0.1163,0.9932,0.4966,0.9932]$} & $e_{32}$ & {$[0.110,0.4036,0.110,0.9938,0.4969,0.9938]$} \\
$e_{13}$ & {$[0.325,0.441,0.1163,0.9932,0.4966,0.9932]$} & $e_{33}$ & {$[0.192,0.4857,0.110,0.9938,0.4969,0.9938]$} \\
$e_{14}$ & {$[0.325,0.1163,0.325,0.9932,0.4966,0.9932]$} & $e_{34}$ & {$[0.192,0.293,0.110,0.9938,0.4969,0.9938]$} \\
$e_{15}$ & {$[0.1163,0.325,0.1163,0.9932,0.4966,0.9932]$} & $e_{35}$ & {$[0.1858,0.4919,0.100,0.9825,0.4912,0.9948]$} \\
$e_{16}$ & {$[0.5,0.093,0.480,0.866,0.433,1.061]$} & $e_{36}$ & {$[0.1858,0.3060,0.100,0.9825,0.4912,0.9948]$} \\
$e_{17}$ & {$[0.5,0.406,0.206,0.866,0.433,1.061]$} & $e_{37}$ & {$[0.272,0.472,0.064,0.962,0.481,0.997]$} \\
$e_{18}$ & {$[0.5,0.5,0.5,0.866,0.172,0.866]$} & $e_{38}$ & {$[0.272,0.199,0.262,0.962,0.481,0.997]$} \\
$e_{19}$ & {$[0.5,0.399,0.240,0.866,0.346,0.9706]$} & $e_{39}$ & {$[0.5,0.3829,0.2397,0.9708,0.4628,0.9708]$}
\end{tabular}

TABLE 4. Numerical expression of points on $(V(I) \cap \partial \mathcal{F}) / \sim \Delta$. 


\begin{tabular}{|c|c|c|c|}
\hline $\mathrm{t}: p$ & maximal label: $L_{p}$ & $\mathrm{pt}: p$ & maximal label: $L_{p}$ \\
\hline$e_{0}$ & {$\left[\mathbf{1}, \mathbf{2}, O, \theta E_{1}, \theta E_{2}, \theta I_{+}, Y_{1}, Y_{3}, X_{1}, X_{2}\right]$} & $\overline{\theta e_{8}}$ & {$\left[O, Y_{1}, Y_{2}, \theta X_{1}, \theta X_{3}, \theta X_{2}\right]$} \\
\hline$\theta_{2} e_{0}$ & {$\left[\mathbf{1}, \mathbf{2}, O, \theta E_{1}, E_{2}, \theta I_{-}, Y_{1}, Y_{3}, X_{1}, \theta X_{2}\right]$} & $e_{9}$ & {$\left[\mathbf{1}, O, Y_{2}, X_{1}, X_{3}, X_{2}\right]$} \\
\hline$\theta_{1} e_{0}$ & {$\left[\mathbf{1}, \mathbf{2}, O, E_{1}, \theta E_{2}, I_{-}, Y_{1}, Y_{3}, X_{2}, \theta X_{1}\right]$} & $\theta_{2} e_{9}$ & {$\left[\mathbf{1}, E_{2}, \theta J_{1}, Y_{2}, X_{1}, X_{3}, \theta X_{2}\right]$} \\
\hline$\theta_{1} \theta_{2} e_{0}$ & {$\left[\mathbf{1}, \mathbf{2}, O, E_{1}, E_{2}, I_{+}, Y_{1}, Y_{3}, \theta X_{1}, \theta X_{2}\right]$} & $\theta_{3} e_{9}$ & {$\left[\mathbf{1}, \theta I_{+}, J, Y_{2}, X_{1}, X_{2}, \theta X_{3}\right]$} \\
\hline$\theta_{3} e_{1}$ & {$\left[\mathbf{1}, \mathbf{2}, \theta R, \theta E_{1}, \theta E_{2}, \theta I_{+}, J, Y_{1}, Y_{2}\right]$} & $\theta_{3} \theta_{2} e_{9}$ & {$\left[\mathbf{1}, \theta E_{1}, J_{2}, Y_{2}, X_{1}, \theta X_{3}, \theta X_{2}\right]$} \\
\hline$\theta_{1} \theta_{2} e_{1}$ & {$\left[\mathbf{1}, \mathbf{2}, R, E_{1}, E_{2}, I_{+}, \theta J, Y_{1}, Y_{2}\right]$} & $\theta_{1} e_{9}$ & {$\left[\mathbf{1}, E_{1}, \theta J_{2}, Y_{2}, X_{3}, X_{2}, \theta X_{1}\right]$} \\
\hline$\theta_{2} e_{2}$ & {$\left[R, E_{2}, \theta J_{1}, Y_{1}, Y_{2}, X_{3}\right]$} & $\theta_{1} \theta_{2} e_{9}$ & {$\left[\mathbf{1}, I_{+}, \theta J, Y_{2}, X_{3}, \theta X_{1}, \theta X_{2}\right]$} \\
\hline$\theta_{3} \theta_{2} e_{2}$ & {$\left[\theta R, \theta E_{1}, J_{2}, Y_{1}, Y_{2}, \theta X_{3}\right]$} & $\theta_{1} \theta_{3} e_{9}$ & {$\left[\mathbf{1}, \theta E_{2}, J_{1}, Y_{2}, X_{2}, \theta X_{1}, \theta X_{3}\right]$} \\
\hline$\theta_{1} e_{2}$ & {$\left[R, E_{1}, \theta J_{2}, Y_{1}, Y_{2}, X_{3}\right]$} & $\theta e_{9}$ & {$\left[\mathbf{1}, O, Y_{2}, \theta X_{1}, \theta X_{3}, \theta X_{2}\right]$} \\
\hline$\theta_{1} \theta_{3} e_{2}$ & {$\left[\theta R, \theta E_{2}, J_{1}, Y_{1}, Y_{2}, \theta X_{3}\right]$} & $e_{10}$ & {$\left[\mathbf{1}, O, \theta J_{1}, Y_{2}, X_{1}, X_{3}\right]$} \\
\hline$e_{3}$ & {$\left[\mathbf{1}, \mathbf{2}, Y_{1}, Y_{3}, X_{1}, X_{3}, X_{2}\right]$} & $\theta_{3} e_{10}$ & {$\left[\mathbf{1}, \theta E_{1}, J, Y_{2}, X_{1}, \theta X_{3}\right]$} \\
\hline$\theta_{2} e_{3}$ & {$\left[\mathbf{1}, \mathbf{2}, Y_{1}, Y_{3}, X_{1}, X_{3}, \theta X_{2}\right]$} & $\theta_{1} e_{10}$ & {$\left[\mathbf{1}, E_{1}, \theta J, Y_{2}, X_{3}, \theta X_{1}\right]$} \\
\hline$\theta_{3} e_{3}$ & {$\left[\mathbf{1}, \mathbf{2}, Y_{1}, Y_{3}, X_{1}, X_{2}, \theta X_{3}\right]$} & $\theta_{1} \theta_{3} e_{10}$ & {$\left[\mathbf{1}, O, J_{1}, Y_{2}, \theta X_{1}, \theta X_{3}\right]$} \\
\hline$\theta_{3} \theta_{2} e_{3}$ & {$\left[1,2, Y_{1}, Y_{3}, X_{1}, \theta X_{3}, \theta X_{2}\right]$} & $e_{11}$ & {$\left[\mathbf{1}, \theta E_{1}, \theta E_{2}, Y_{2}, X_{1}, X_{2}\right]$} \\
\hline$\theta_{1} e_{3}$ & {$\left[\mathbf{1}, \mathbf{2}, Y_{1}, Y_{3}, X_{3}, X_{2}, \theta X_{1}\right]$} & $\theta_{2} e_{11}$ & {$\left[\mathbf{1}, O, \theta I_{-}, Y_{2}, X_{1}, \theta X_{2}\right]$} \\
\hline$\theta_{1} \theta_{2} e_{3}$ & {$\left[\mathbf{1}, \mathbf{2}, Y_{1}, Y_{3}, X_{3}, \theta X_{1}, \theta X_{2}\right]$} & $\theta_{1} e_{11}$ & {$\left[\mathbf{1}, O, I_{-}, Y_{2}, X_{2}, \theta X_{1}\right]$} \\
\hline$\theta_{1} \theta_{3} e_{3}$ & {$\left[\mathbf{1}, \mathbf{2}, Y_{1}, Y_{3}, X_{2}, \theta X_{1}, \theta X_{3}\right]$} & $\theta_{1} \theta_{2} e_{11}$ & {$\left[\mathbf{1}, E_{1}, E_{2}, Y_{2}, \theta X_{1}, \theta X_{2}\right]$} \\
\hline$\theta e_{3}$ & {$\left[\mathbf{1}, \mathbf{2}, Y_{1}, Y_{3}, \theta X_{1}, \theta X_{3}, \theta X_{2}\right]$} & $\theta_{2} e_{12}$ & {$\left[\mathbf{1}, \mathbf{2}, R, E_{2}, Y_{1}, Y_{2}\right]$} \\
\hline$e_{4}$ & {$\left[\mathbf{2}, O, \theta J_{1}, Y_{1}, Y_{2}, X_{1}, X_{3}\right]$} & $\theta_{3} \theta_{2} e_{12}$ & {$\left[\mathbf{1}, \mathbf{2}, \theta R, \theta E_{1}, Y_{1}, Y_{2}\right]$} \\
\hline$\theta_{2} \sigma_{0} e_{4}$ & {$\left[\mathbf{1}, E_{2}, \theta J, Y_{1}, Y_{2}, X_{3}, \theta X_{2}\right]$} & $\theta_{1} e_{12}$ & {$\left[\mathbf{1}, \mathbf{2}, R, E_{1}, Y_{1}, Y_{2}\right]$} \\
\hline$\theta_{3} e_{4}$ & {$\left[\mathbf{2}, \theta E_{1}, J, Y_{1}, Y_{2}, X_{1}, \theta X_{3}\right]$} & $\theta_{1} \theta_{3} e_{12}$ & {$\left[\mathbf{1}, \mathbf{2}, \theta R, \theta E_{2}, Y_{1}, Y_{2}\right]$} \\
\hline$\theta_{3} \theta_{2} \sigma_{0} e_{4}$ & {$\left[\mathbf{1}, O, J_{2}, Y_{1}, Y_{2}, \theta X_{3}, \theta X_{2}\right]$} & $\theta_{2} e_{13}$ & {$\left[\mathbf{2}, O, E_{2}, \theta J_{1}, Y_{1}, Y_{2}\right]$} \\
\hline$\theta_{3} \sigma_{0} e_{4}$ & {$\left[\mathbf{1}, \theta E_{2}, J, Y_{1}, Y_{2}, X_{2}, \theta X_{3}\right]$} & $\theta_{3} \theta_{2} \sigma_{0} e_{13}$ & {$\left[\mathbf{1}, O, \theta E_{1}, J_{2}, Y_{1}, Y_{2}\right]$} \\
\hline$\theta_{1} e_{4}$ & {$\left[\mathbf{2}, E_{1}, \theta J, Y_{1}, Y_{2}, X_{3}, \theta X_{1}\right]$} & $\theta_{1} \theta_{3} e_{13}$ & {$\left[2, O, \theta E_{2}, J_{1}, Y_{1}, Y_{2}\right]$} \\
\hline$\theta_{1} \theta_{3} e_{4}$ & {$\left[\mathbf{2}, O, J_{1}, Y_{1}, Y_{2}, \theta X_{1}, \theta X_{3}\right]$} & $\theta_{1} \sigma_{0} e_{13}$ & {$\left[\mathbf{1}, O, E_{1}, \theta J_{2}, Y_{1}, Y_{2}\right]$} \\
\hline$\sigma_{0} e_{4}$ & {$\left[\mathbf{1}, O, \theta J_{2}, Y_{1}, Y_{2}, X_{3}, X_{2}\right]$} & $\theta_{3} e_{14}$ & {$\left[\theta R, O, \theta E_{1}, \theta E_{2}, Y_{1}, Y_{2}\right]$} \\
\hline$e_{5}$ & {$\left[\theta R, \theta E_{1}, \theta E_{2}, Y_{1}, Y_{2}, X_{1}, X_{2}\right]$} & $\theta_{1} \theta_{2} e_{14}$ & {$\left[R, O, E_{1}, E_{2}, Y_{1}, Y_{2}\right]$} \\
\hline$\theta_{1} \theta_{2} e_{5}$ & {$\left[R, E_{1}, E_{2}, Y_{1}, Y_{2}, \theta X_{1}, \theta X_{2}\right]$} & $\theta_{3} e_{15}$ & {$\left[\mathbf{1}, \mathbf{2}, \theta R, O, Y_{1}, Y_{2}\right]$} \\
\hline$e_{6}$ & {$\left[\mathbf{1}, \mathbf{2}, R, Y_{1}, Y_{2}, X_{3}\right]$} & $\theta_{1} \theta_{2} e_{15}$ & {$\left[\mathbf{1}, \mathbf{2}, R, O, Y_{1}, Y_{2}\right]$} \\
\hline$\theta_{3} e_{6}$ & {$\left[\mathbf{1}, \mathbf{2}, \theta R, Y_{1}, Y_{2}, \theta X_{3}\right]$} & $e_{16}$ & {$\left[\mathbf{1}, O, \theta E_{1}, \theta E_{2}, Y_{2}, X_{1}\right]$} \\
\hline$e_{7}$ & {$\left[\mathbf{1}, O, \theta E_{2}, Y_{2}, X_{1}, X_{2}\right]$} & $\theta_{3} \theta_{2} e_{16}$ & {$\left[\mathbf{1}, O, \theta E_{1}, \theta I_{-}, Y_{2}, X_{1}\right]$} \\
\hline$\theta_{2} e_{7}$ & {$\left[\mathbf{1}, O, E_{2}, Y_{2}, X_{1}, \theta X_{2}\right]$} & $\theta_{1} e_{16}$ & {$\left[\mathbf{1}, O, E_{1}, I_{-}, Y_{2}, \theta X_{1}\right]$} \\
\hline$\theta_{3} e_{7}$ & {$\left[\mathbf{1}, \theta E_{1}, \theta I_{+}, Y_{2}, X_{1}, X_{2}\right]$} & $\theta e_{16}$ & {$\left[\mathbf{1}, O, E_{1}, E_{2}, Y_{2}, \theta X_{1}\right]$} \\
\hline$\theta_{3} \theta_{2} e_{7}$ & {$\left[\mathbf{1}, \theta E_{1}, \theta I_{-}, Y_{2}, X_{1}, \theta X_{2}\right]$} & $\theta_{2} e_{17}$ & {$\left[\mathbf{1}, O, E_{2}, \theta J_{1}, Y_{2}, X_{1}\right]$} \\
\hline$\theta_{1} e_{7}$ & {$\left[\mathbf{1}, E_{1}, I_{-}, Y_{2}, X_{2}, \theta X_{1}\right]$} & $\theta_{3} e_{17}$ & {$\left[\mathbf{1}, \theta E_{1}, \theta I_{+}, J, Y_{2}, X_{1}\right]$} \\
\hline$\theta_{1} \theta_{2} e_{7}$ & {$\left[\mathbf{1}, E_{1}, I_{+}, Y_{2}, \theta X_{1}, \theta X_{2}\right]$} & $\theta_{1} \theta_{2} e_{17}$ & {$\left[\mathbf{1}, E_{1}, I_{+}, \theta J, Y_{2}, \theta X_{1}\right]$} \\
\hline$\theta_{1} \theta_{3} e_{7}$ & {$\left[\mathbf{1}, O, \theta E_{2}, Y_{2}, X_{2}, \theta X_{1}\right]$} & $\theta_{1} \theta_{3} e_{17}$ & {$\left[\mathbf{1}, O, \theta E_{2}, J_{1}, Y_{2}, \theta X_{1}\right]$} \\
\hline$\theta e_{7}$ & {$\left[\mathbf{1}, O, E_{2}, Y_{2}, \theta X_{1}, \theta X_{2}\right]$} & $e_{18}$ & {$\left[\mathbf{1}, \mathbf{2}, O, Y_{1}, X_{1}, X_{3}, X_{2}\right]$} \\
\hline$e_{8}$ & {$\left[O, Y_{1}, Y_{2}, X_{1}, X_{3}, X_{2}\right]$} & $\theta_{2} e_{18}$ & {$\left[\mathbf{1}, \mathbf{2}, E_{2}, \theta J_{1}, Y_{1}, X_{1}, X_{3}, \theta X_{2}\right]$} \\
\hline$\theta_{2} e_{8}$ & {$\left[E_{2}, \theta J_{1}, Y_{1}, Y_{2}, X_{1}, X_{3}, \theta X_{2}\right]$} & $\theta_{3} e_{18}$ & {$\left[\mathbf{1}, \mathbf{2}, \theta I_{+}, J, Y_{1}, X_{1}, X_{2}, \theta X_{3}\right]$} \\
\hline$\theta_{3} e_{8}$ & {$\left[\theta I_{+}, J, Y_{1}, Y_{2}, X_{1}, X_{2}, \theta X_{3}\right]$} & $\theta_{3} \theta_{2} e_{18}$ & {$\left[\mathbf{1}, \mathbf{2}, \theta E_{1}, J_{2}, Y_{1}, X_{1}, \theta X_{3}, \theta X_{2}\right]$} \\
\hline$\theta_{3} \theta_{2} e_{8}$ & {$\left[\theta E_{1}, J_{2}, Y_{1}, Y_{2}, X_{1}, \theta X_{3}, \theta X_{2}\right]$} & $\theta_{1} e_{18}$ & {$\left[\mathbf{1}, \mathbf{2}, E_{1}, \theta J_{2}, Y_{1}, X_{3}, X_{2}, \theta X_{1}\right]$} \\
\hline$\theta_{1} e_{8}$ & {$\left[E_{1}, \theta J_{2}, Y_{1}, Y_{2}, X_{3}, X_{2}, \theta X_{1}\right]$} & $\theta_{1} \theta_{2} e_{18}$ & {$\left[\mathbf{1}, \mathbf{2}, I_{+}, \theta J, Y_{1}, X_{3}, \theta X_{1}, \theta X_{2}\right]$} \\
\hline$\theta_{1} \theta_{2} e_{8}$ & {$\left[I_{+}, \theta J, Y_{1}, Y_{2}, X_{3}, \theta X_{1}, \theta X_{2}\right]$} & $\theta_{1} \theta_{3} e_{18}$ & {$\left[\mathbf{1}, \mathbf{2}, \theta E_{2}, J_{1}, Y_{1}, X_{2}, \theta X_{1}, \theta X_{3}\right]$} \\
\hline$\theta_{1} \theta_{3} e_{8}$ & {$\left[\theta E_{2}, J_{1}, Y_{1}, Y_{2}, X_{2}, \theta X_{1}, \theta X_{3}\right]$} & $\theta e_{18}$ & {$\left[\mathbf{1}, \mathbf{2}, O, Y_{1}, \theta X_{1}, \theta X_{3}, \theta X_{2}\right]$} \\
\hline
\end{tabular}

TABLE 5. Points $p$ and its associated label $L_{p} \subset \Lambda$.

Proposition 6.5. Let $G \subset I$ be a Gröbner basis of I with respect to the lexicographic order $X_{1} \succ X_{2} \succ \cdots \succ X_{n}$. Then
If $G$ is reduced, then $G \cap \mathbb{Q}\left[X_{n}\right]=m\left(I, X_{n} ; t\right)$.

One way to obtain the minimal polynomials is to compute Gröbner bases. Finally, we refer to the so-called shape basis. 


\begin{tabular}{|c|c|c|c|}
\hline pt: $p$ & maximal label: $L_{p}$ & $\mathrm{pt}: p$ & maximal label: $L_{p}$ \\
\hline$\overline{\theta_{2} e_{19}}$ & {$\left[\mathbf{1}, \mathbf{2}, O, E_{2}, \theta J_{1}, X_{1}\right]$} & $\overline{\theta_{1} \theta_{2} e_{27}}$ & {$\left[\mathbf{1}, E_{1}, E_{2}, I_{+}, Y_{2}, \theta X_{2}\right]$} \\
\hline$\theta_{3} e_{19}$ & {$\left[\mathbf{1}, \mathbf{2}, \theta E_{1}, \theta I_{+}, J, X_{1}\right]$} & $\theta_{2} e_{28}$ & {$\left[\mathbf{1}, \mathbf{2}, O, \theta J_{1}, Y_{2}, X_{3}\right]$} \\
\hline$\theta_{1} \theta_{2} e_{19}$ & {$\left[\mathbf{1}, \mathbf{2}, E_{1}, I_{+}, \theta J, \theta X_{1}\right]$} & $\theta_{3} \theta_{2} e_{28}$ & {$\left[\mathbf{1}, \mathbf{2}, \theta E_{1}, J, Y_{2}, \theta X_{3}\right]$} \\
\hline$\theta_{1} \theta_{3} e_{19}$ & {$\left[\mathbf{1}, \mathbf{2}, O, \theta E_{2}, J_{1}, \theta X_{1}\right]$} & $\theta_{1} e_{28}$ & {$\left[\mathbf{1}, \mathbf{2}, E_{1}, \theta J, Y_{2}, X_{3}\right]$} \\
\hline$\theta_{3} e_{20}$ & {$\left[\mathbf{1}, \mathbf{2}, \theta R, O, \theta E_{1}, \theta E_{2}, Y_{1}\right]$} & $\theta_{1} \theta_{3} e_{28}$ & {$\left[\mathbf{1}, \mathbf{2}, O, J_{1}, Y_{2}, \theta X_{3}\right]$} \\
\hline$\theta_{1} \theta_{2} e_{20}$ & {$\left[\mathbf{1}, \mathbf{2}, R, O, E_{1}, E_{2}, Y_{1}\right]$} & $e_{29}$ & {$\left[\mathbf{1}, \mathbf{2}, O, \theta J_{1}, X_{1}, X_{3}\right]$} \\
\hline$\theta_{2} e_{21}$ & {$\left[\mathbf{2}, R, \theta J_{1}, Y_{1}, Y_{2}, X_{3}\right]$} & $\theta_{3} e_{29}$ & {$\left[\mathbf{1}, \mathbf{2}, \theta E_{1}, J, X_{1}, \theta X_{3}\right]$} \\
\hline$\theta_{2} \sigma_{0} e_{21}$ & {$\left[\mathbf{1}, R, E_{2}, Y_{1}, Y_{2}, X_{3}\right]$} & $\theta_{1} e_{29}$ & {$\left[\mathbf{1}, \mathbf{2}, E_{1}, \theta J, X_{3}, \theta X_{1}\right]$} \\
\hline$\theta_{3} \theta_{2} e_{21}$ & {$\left[\mathbf{2}, \theta R, \theta E_{1}, Y_{1}, Y_{2}, \theta X_{3}\right]$} & $\theta_{1} \theta_{3} e_{29}$ & {$\left[\mathbf{1}, \mathbf{2}, O, J_{1}, \theta X_{1}, \theta X_{3}\right]$} \\
\hline$\theta_{3} \theta_{2} \sigma_{0} e_{21}$ & {$\left[\mathbf{1}, \theta R, J_{2}, Y_{1}, Y_{2}, \theta X_{3}\right]$} & $\theta_{2} e_{30}$ & {$\left[\mathbf{1}, \mathbf{2}, R, E_{2}, \theta J_{1}, Y_{1}, X_{3}\right]$} \\
\hline$\theta_{1} e_{21}$ & {$\left[2, R, E_{1}, Y_{1}, Y_{2}, X_{3}\right]$} & $\theta_{3} \theta_{2} e_{30}$ & {$\left[\mathbf{1}, \mathbf{2}, \theta R, \theta E_{1}, J_{2}, Y_{1}, \theta X_{3}\right]$} \\
\hline$\theta_{1} \theta_{3} e_{21}$ & {$\left[\mathbf{2}, \theta R, J_{1}, Y_{1}, Y_{2}, \theta X_{3}\right]$} & $\theta_{1} e_{30}$ & {$\left[\mathbf{1}, \mathbf{2}, R, E_{1}, \theta J_{2}, Y_{1}, X_{3}\right]$} \\
\hline$\theta_{1} \theta_{3} \sigma_{0} e_{21}$ & {$\left[\mathbf{1}, \theta R, \theta E_{2}, Y_{1}, Y_{2}, \theta X_{3}\right]$} & $\theta_{1} \theta_{3} e_{30}$ & {$\left[\mathbf{1}, \mathbf{2}, \theta R, \theta E_{2}, J_{1}, Y_{1}, \theta X_{3}\right]$} \\
\hline$\theta_{1} \sigma_{0} e_{21}$ & {$\left[\mathbf{1}, R, \theta J_{2}, Y_{1}, Y_{2}, X_{3}\right]$} & $\theta_{2} e_{31}$ & {$\left[\mathbf{1}, \mathbf{2}, R, \theta J_{1}, Y_{2}, X_{3}\right]$} \\
\hline$\theta_{2} e_{22}$ & {$\left[\mathbf{1}, R, E_{2}, \theta J_{1}, Y_{2}, X_{3}\right]$} & $\theta_{3} \theta_{2} e_{31}$ & {$\left[\mathbf{1}, \mathbf{2}, \theta R, \theta E_{1}, Y_{2}, \theta X_{3}\right]$} \\
\hline$\theta_{3} \theta_{2} e_{22}$ & {$\left[\mathbf{1}, \theta R, \theta E_{1}, J_{2}, Y_{2}, \theta X_{3}\right]$} & $\theta_{1} e_{31}$ & {$\left[\mathbf{1}, \mathbf{2}, R, E_{1}, Y_{2}, X_{3}\right]$} \\
\hline$\theta_{1} e_{22}$ & {$\left[\mathbf{1}, R, E_{1}, \theta J_{2}, Y_{2}, X_{3}\right]$} & $\theta_{1} \theta_{3} e_{31}$ & {$\left[\mathbf{1}, \mathbf{2}, \theta R, J_{1}, Y_{2}, \theta X_{3}\right]$} \\
\hline$\theta_{1} \theta_{3} e_{22}$ & {$\left[\mathbf{1}, \theta R, \theta E_{2}, J_{1}, Y_{2}, \theta X_{3}\right]$} & $\theta_{2} e_{32}$ & {$\left[\mathbf{1}, \mathbf{2}, O, E_{2}, Y_{1}, Y_{2}\right]$} \\
\hline$e_{23}$ & {$\left[\mathbf{1}, \mathbf{2}, O, Y_{1}, Y_{2}, X_{3}\right]$} & $\theta_{3} \theta_{2} e_{32}$ & {$\left[\mathbf{1}, \mathbf{2}, O, \theta E_{1}, Y_{1}, Y_{2}\right]$} \\
\hline$\theta_{3} e_{23}$ & {$\left[\mathbf{1}, \mathbf{2}, J, Y_{1}, Y_{2}, \theta X_{3}\right]$} & $\theta_{1} e_{32}$ & {$\left[\mathbf{1}, \mathbf{2}, O, E_{1}, Y_{1}, Y_{2}\right]$} \\
\hline$\theta_{1} \theta_{2} e_{23}$ & {$\left[\mathbf{1}, \mathbf{2}, \theta J, Y_{1}, Y_{2}, X_{3}\right]$} & $\theta_{1} \theta_{3} e_{32}$ & {$\left[\mathbf{1}, \mathbf{2}, O, \theta E_{2}, Y_{1}, Y_{2}\right]$} \\
\hline$\theta e_{23}$ & {$\left[\mathbf{1}, \mathbf{2}, O, Y_{1}, Y_{2}, \theta X_{3}\right]$} & $\theta_{2} e_{33}$ & {$\left[\mathbf{2}, R, E_{2}, \theta J_{1}, Y_{1}, Y_{2}\right]$} \\
\hline$\theta_{3} e_{24}$ & {$\left[\mathbf{1}, \theta E_{2}, \theta I_{+}, J, Y_{2}, X_{2}\right]$} & $\theta_{3} \theta_{2} \sigma_{0} e_{33}$ & {$\left[\mathbf{1}, \theta R, \theta E_{1}, J_{2}, Y_{1}, Y_{2}\right]$} \\
\hline$\theta_{3} \theta_{2} e_{24}$ & {$\left[\mathbf{1}, O, \theta E_{1}, J_{2}, Y_{2}, \theta X_{2}\right]$} & $\theta_{1} \theta_{3} e_{33}$ & {$\left[\mathbf{2}, \theta R, \theta E_{2}, J_{1}, Y_{1}, Y_{2}\right]$} \\
\hline$\theta_{1} e_{24}$ & {$\left[\mathbf{1}, O, E_{1}, \theta J_{2}, Y_{2}, X_{2}\right]$} & $\theta_{1} \sigma_{0} e_{33}$ & {$\left[\mathbf{1}, R, E_{1}, \theta J_{2}, Y_{1}, Y_{2}\right]$} \\
\hline$\theta_{1} \theta_{2} e_{24}$ & {$\left[\mathbf{1}, E_{2}, I_{+}, \theta J, Y_{2}, \theta X_{2}\right]$} & $\theta_{3} e_{34}$ & {$\left[2, \theta R, O, \theta E_{1}, Y_{1}, Y_{2}\right]$} \\
\hline$e_{25}$ & {$\left[O, \theta E_{1}, \theta E_{2}, Y_{1}, Y_{2}, X_{1}, X_{2}\right]$} & $\theta_{3} \sigma_{0} e_{34}$ & {$\left[\mathbf{1}, \theta R, O, \theta E_{2}, Y_{1}, Y_{2}\right]$} \\
\hline$\theta_{2} e_{25}$ & {$\left[O, E_{2}, \theta I_{-}, Y_{1}, Y_{2}, X_{1}, \theta X_{2}\right]$} & $\theta_{1} \theta_{2} e_{34}$ & {$\left[\mathbf{2}, R, O, E_{1}, Y_{1}, Y_{2}\right]$} \\
\hline$\theta_{3} e_{25}$ & {$\left[\theta E_{1}, \theta E_{2}, \theta I_{+}, Y_{1}, Y_{2}, X_{1}, X_{2}\right]$} & $\theta_{1} \theta_{2} \sigma_{0} e_{34}$ & {$\left[\mathbf{1}, R, O, E_{2}, Y_{1}, Y_{2}\right]$} \\
\hline$\theta_{3} \theta_{2} e_{25}$ & {$\left[O, \theta E_{1}, \theta I_{-}, Y_{1}, Y_{2}, X_{1}, \theta X_{2}\right]$} & $\theta_{2} e_{35}$ & {$\left[\mathbf{1}, \mathbf{2}, R, E_{2}, \theta J_{1}, Y_{2}\right]$} \\
\hline$\theta_{1} e_{25}$ & {$\left[O, E_{1}, I_{-}, Y_{1}, Y_{2}, X_{2}, \theta X_{1}\right]$} & $\theta_{1} \theta_{3} e_{35}$ & {$\left[\mathbf{1}, \mathbf{2}, \theta R, \theta E_{2}, J_{1}, Y_{2}\right]$} \\
\hline$\theta_{1} \theta_{2} e_{25}$ & {$\left[E_{1}, E_{2}, I_{+}, Y_{1}, Y_{2}, \theta X_{1}, \theta X_{2}\right]$} & $\theta_{3} e_{36}$ & {$\left[\mathbf{1}, \mathbf{2}, \theta R, O, \theta E_{1}, Y_{2}\right]$} \\
\hline$\theta_{1} \theta_{3} e_{25}$ & {$\left[O, \theta E_{2}, I_{-}, Y_{1}, Y_{2}, X_{2}, \theta X_{1}\right]$} & $\theta_{1} \theta_{2} e_{36}$ & {$\left[\mathbf{1}, \mathbf{2}, R, O, E_{1}, Y_{2}\right]$} \\
\hline$\theta e_{25}$ & {$\left[O, E_{1}, E_{2}, Y_{1}, Y_{2}, \theta X_{1}, \theta X_{2}\right]$} & $\theta_{2} e_{37}$ & {$\left[\mathbf{1}, \mathbf{2}, O, E_{2}, \theta J_{1}, Y_{2}\right]$} \\
\hline$\theta_{2} e_{26}$ & {$\left[O, E_{2}, \theta J_{1}, Y_{1}, Y_{2}, X_{1}\right]$} & $\theta_{1} \theta_{3} e_{37}$ & {$\left[\mathbf{1}, \mathbf{2}, O, \theta E_{2}, J_{1}, Y_{2}\right]$} \\
\hline$\theta_{3} e_{26}$ & {$\left[\theta E_{1}, \theta I_{+}, J, Y_{1}, Y_{2}, X_{1}\right]$} & $\theta_{3} e_{38}$ & {$\left[\mathbf{1}, \theta R, O, \theta E_{1}, \theta E_{2}, Y_{2}\right]$} \\
\hline$\theta_{3} \theta_{2} \sigma_{0} e_{26}$ & {$\left[O, \theta E_{1}, J_{2}, Y_{1}, Y_{2}, \theta X_{2}\right]$} & $\theta_{1} \theta_{2} e_{38}$ & {$\left[\mathbf{1}, R, O, E_{1}, E_{2}, Y_{2}\right]$} \\
\hline$\theta_{3} \sigma_{0} e_{26}$ & {$\left[\theta E_{2}, \theta I_{+}, J, Y_{1}, Y_{2}, X_{2}\right]$} & $\theta_{2} e_{39}$ & {$\left[2, O, E_{2}, \theta J_{1}, Y_{1}, X_{1}\right]$} \\
\hline$\theta_{1} \theta_{2} e_{26}$ & {$\left[E_{1}, I_{+}, \theta J, Y_{1}, Y_{2}, \theta X_{1}\right]$} & $\theta_{3} e_{39}$ & {$\left[2, \theta E_{1}, \theta I_{+}, J, Y_{1}, X_{1}\right]$} \\
\hline$\theta_{1} \theta_{2} \sigma_{0} e_{26}$ & {$\left[E_{2}, I_{+}, \theta J, Y_{1}, Y_{2}, \theta X_{2}\right]$} & $\theta_{3} \theta_{2} \sigma_{0} e_{39}$ & {$\left[\mathbf{1}, O, \theta E_{1}, J_{2}, Y_{1}, \theta X_{2}\right]$} \\
\hline$\theta_{1} \theta_{3} e_{26}$ & {$\left[O, \theta E_{2}, J_{1}, Y_{1}, Y_{2}, \theta X_{1}\right]$} & $\theta_{3} \sigma_{0} e_{39}$ & {$\left[\mathbf{1}, \theta E_{2}, \theta I_{+}, J, Y_{1}, X_{2}\right]$} \\
\hline$\theta_{1} \sigma_{0} e_{26}$ & {$\left[O, E_{1}, \theta J_{2}, Y_{1}, Y_{2}, X_{2}\right]$} & $\theta_{1} \theta_{2} e_{39}$ & {$\left[2, E_{1}, I_{+}, \theta J, Y_{1}, \theta X_{1}\right]$} \\
\hline$\theta_{3} e_{27}$ & {$\left[\mathbf{1}, \theta E_{1}, \theta E_{2}, \theta I_{+}, Y_{2}, X_{2}\right]$} & $\theta_{1} \theta_{2} \sigma_{0} e_{39}$ & {$\left[\mathbf{1}, E_{2}, I_{+}, \theta J, Y_{1}, \theta X_{2}\right]$} \\
\hline$\theta_{3} \theta_{2} e_{27}$ & {$\left[\mathbf{1}, O, \theta E_{1}, \theta I_{-}, Y_{2}, \theta X_{2}\right]$} & $\theta_{1} \theta_{3} e_{39}$ & {$\left[2, O, \theta E_{2}, J_{1}, Y_{1}, \theta X_{1}\right]$} \\
\hline$\theta_{1} e_{27}$ & {$\left[\mathbf{1}, O, E_{1}, I_{-}, Y_{2}, X_{2}\right]$} & $\theta_{1} \sigma_{0} e_{39}$ & {$\left[\mathbf{1}, O, E_{1}, \theta J_{2}, Y_{1}, X_{2}\right]$} \\
\hline
\end{tabular}

TABLE 6. Points $p$ and their associated labels $L_{p} \subset \Lambda$ (2).

Proposition 6.6. Suppose that $I$ is zero-dimensional and that $\operatorname{dim} P / I=\operatorname{deg}\left(m\left(I, X_{n} ; t\right)\right)$. Then there is a set of polynomials $\left\{g_{i}(t)\right\}$ such that the Gröbner basis with re- spect to the lexicographic order is

$$
\begin{aligned}
G=\{ & X_{1}-g_{1}\left(X_{n}\right), X_{2}-g_{2}\left(X_{n}\right), \ldots, \\
& \left.X_{n-1}-g_{n-1}\left(X_{n}\right), m\left(I, X_{n} ; t\right)\right\} .
\end{aligned}
$$




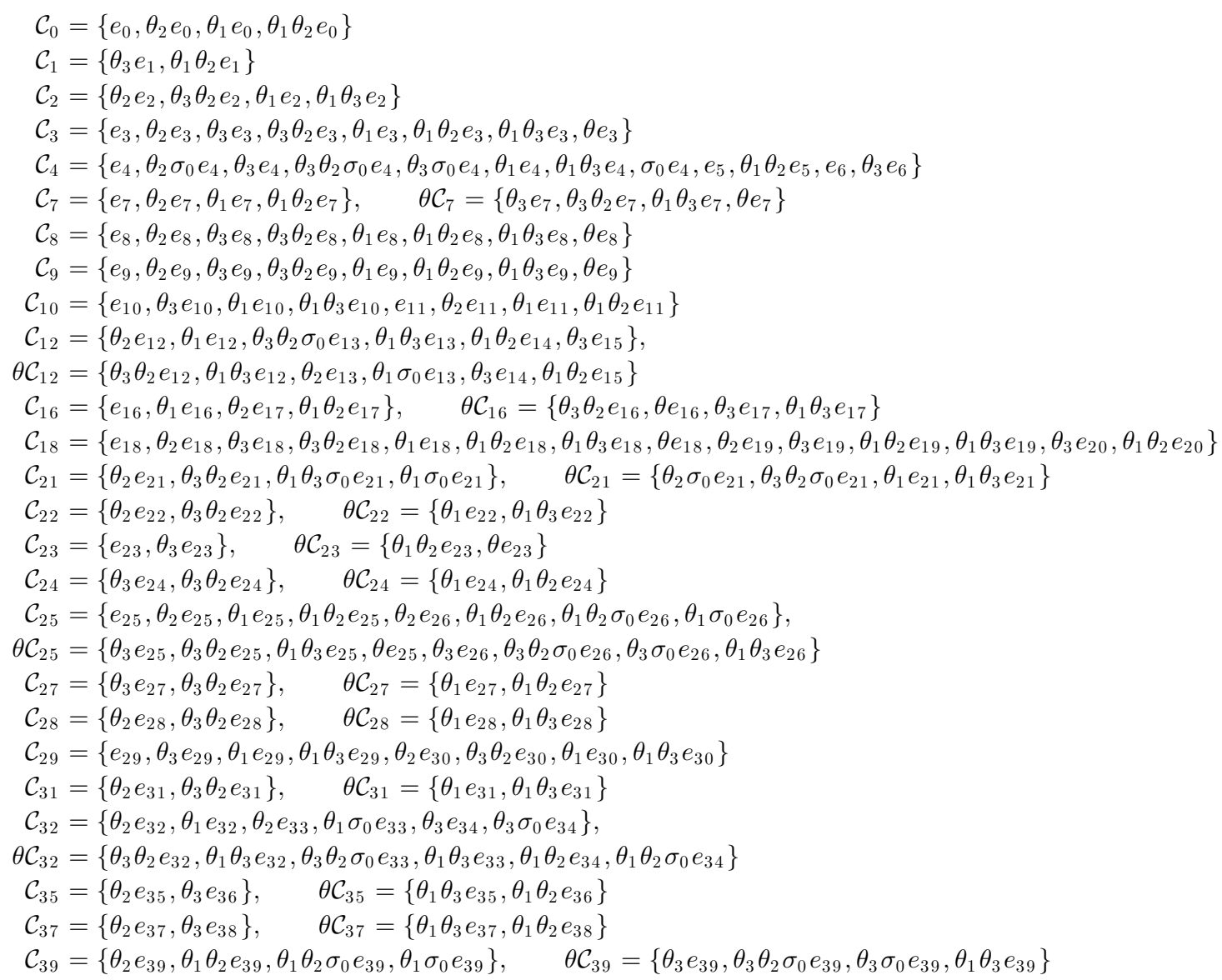

TABLE 7. $\Gamma$-equivalence sets $\mathcal{C}_{k}$ and $\theta \mathcal{C}_{k}$.

So the problem is reduced to obtaining the zeros of the univariate polynomial $m\left(I, X_{n} ; t\right)$. The other coordinates of the zeros are obtained as certain values of the univariate polynomial.

The real roots of a one-variable polynomial are fairly easily obtained by Newton's method. So by the proposition above, we obtain all the real zero points of $V(I)$ when $I$ is zero-dimensional.

Returning to our case, the computation of a Gröbner basis with respect to the lexicographic order is sometimes too expensive. In this unfortunate situation, we do a mixed strategy utilizing other kinds of term order. We divide step 2 into the following:

step 2-1. Compute the minimal polynomials $m\left(I, X_{i j} ; t\right), m\left(I, Y_{i j} ; t\right)$ and their real roots.

step 2-2. Check $p \in V(I)$ using floating-point computation up to a certain accuracy for all combinations of real roots of the minimal polynomials.
In the system ASIR, this can be done by the command minipoly. The real roots are obtained by Newton's method.

To speed up the search, we apply the following pruning. We can skip to the next $L$ if a root violates condition (1) or (2) in Section 2.

\subsection{Step 4: Nonempty 0-Cells}

We know the points in $V_{0}$ of all 0-dimensional ideals $I_{L}$ by step 2 . Then step 3 can be easily carried out by the inclusion-reversing property. In step 4 , we decide whether $p \in V_{0}$ is in $W_{L}$. This can be done by a positivity check for $f_{\lambda} \lambda \in \Lambda \backslash L$ by a floating-point computation in view of step 3 ; if a point can attain zero on $f_{\lambda}, \lambda \notin L$, this would violate the maximality of $L$.

Search Result 6.7. Among the zero-dimensional ideals $I_{L}$ there are 2146 labels $L$, so that $W_{L}$ is nonempty. In fact, one has $\left|W_{L}\right|=\left|V\left(I_{L}\right) \cap \partial \mathcal{F}_{2}\right| \leq 2$. 
Search Result 6.8. These 2146 labels produce 180 points on the boundary of the fundamental domain $V\left(I_{L}\right) \cap \partial \mathcal{F}$ indicated in Table 5.

Our search results suggest that Theorem 5.1 is best possible.

\section{PROOF OF THEOREM 5.1}

Once the point $p$ is explicitly given, to show that the point satisfies the positivity $f_{\lambda}>0, \lambda \in \Lambda \backslash L$, is straightforward. So the remaining part of of the proof of Theorem 5.1 is to show that $p \in V\left(I_{L}\right)_{\mathbb{R}}$. This is achieved through a point-by-point investigation, as the following example indicates.

With the result in Section 6.3 in mind, we revisit Procedure 6.1 for the case $p \stackrel{\Delta}{\sim} e_{12}$. This also clarifies the situation of maximality of labels.

Take $L=\left[\mathbf{1}, \mathbf{2}, \theta R, \theta E_{1}, Y_{1}, Y_{2}\right]$. Then $I_{L}$ can be checked to be zero-dimensional. The minimal polynomial $m(t)=m\left(I, X_{22} ; t\right)$ of $X_{22}$ is of degree 8. By factorization, $X_{22}$ should be $1 / 3, \pm 1 / 5$, or $\Omega_{2}\left(-X_{22}\right)=0$, so $X_{11}=1 / 3, \pm 1 / 5$, or $-\omega_{2}$ as real zeros whose absolute values are less than $1 / 2$.

Consider the case $X_{22}=-\omega_{2}$. Since variables other than $X_{12}$ depend on $X_{22}$ very simply, what is essential is the dependency on $X_{12}$. The shape basis says that

$$
\begin{aligned}
& -40411800 X_{22}^{7}+2571225 X_{22}^{6}+91990172 X_{22}^{5} \\
& \quad+52140601 X_{22}^{4}-53576848 X_{22}^{3}-50659293 X_{22}^{2} \\
& \quad+16986764 X_{22}-14988288 X_{12}-4052533=0 .
\end{aligned}
$$

Taking this modulo $\Omega_{2}\left(-X_{22}\right)$, we obtain the value of $X_{12}$. In fact, taking the basis corresponding to the graded reverse lexicographical order (grrevlex), we may see the basis would show a simpler dependency: $X_{12}=$ $-\left(1-\omega_{2}\right) / 2$. In any event, we can obtain $\theta_{3} \theta_{2} e_{12}$. After checking positivity, we conclude that $W_{L}$ is nonempty. If $X_{22}= \pm 1 / 5$, this also gives rise to the point $Z \in V\left(I_{L}\right)$, but it would make $f_{O}(Z)$ negative, so such is not the case. On the other hand, when $X_{22}=1 / 3$, it also gives a valid point $\theta_{3} e_{1} \in W_{L}$. So $\left|W_{L}\right|=2$. It turns out that the label $L$ is the maximal label of $\theta_{3} \theta_{2} e_{12}$. Consider the label $L^{\prime}=\left[\mathbf{1}, \mathbf{2}, \theta R, \theta E_{1}, \theta E_{2}, \theta I_{+}, J, Y_{1}, Y_{2}\right]$. Then $L \subset L^{\prime}$ and $W_{L^{\prime}}=\left\{\theta_{3} e_{1}\right\}$. This implies that the maximal label of $\theta_{3} e_{1}$ is $L^{\prime}$.

\section{8. $\quad \boldsymbol{\Gamma}$-EQUIVALENCE CLASSES OF ZERO CELLS}

In this section we consider the $\Gamma$-equivalence classes between the 1800 -cells obtained in the previous sections. Define 40 sets $\mathcal{C}_{k}$ and their $\theta$-images as in Table 7 . We remark that when $k=0,1,2,3,4,8,9,10,18,29$, then $\mathcal{C}_{k}$ is $\theta$-stable, that is, $\mathcal{C}_{k}=\theta \mathcal{C}_{k}$. We discuss $\Gamma$-equivalence and $\Gamma$-inequivalence of the $\mathcal{C}_{k}$ 's.

\subsection{Equivalence Property of $\mathcal{C}_{\boldsymbol{k}}$}

We start with a few lemmas.

Lemma 8.1. $A$ set $\mathcal{C}$ is $\Gamma$-equivalent if and only if $\theta \mathcal{C}$ is.

Proof. If we put $\left(\left(\begin{array}{cc}A & B \\ C & D\end{array}\right)\right)^{\theta}=\left(\begin{array}{cc}A & -B \\ -C & D\end{array}\right)$, then the result follows by $\theta \gamma Z=\gamma^{\theta} \theta Z$.

Lemma 8.2. The sets $\mathcal{C}_{k}, \theta \mathcal{C}_{k}$ for $k=0,2,3,7,8,9$, 21, 22, 23, 24, 27, 28, 31,39 are $\Gamma$-equivalent sets.

Proof. The assertion is clear, because the equivalence inside $\mathcal{C}_{k}$ or $\theta \mathcal{C}_{k}$ can be found by $\sigma_{0}$ and the translations of the $X_{i j}$-coordinates by \pm 1 .

To show the other equivalence property, we first define

$$
\sigma_{1}^{ \pm}=\left(\begin{array}{cc}
I_{-}+E_{21} & \pm E_{2} \\
0 & I_{-}+E_{12}
\end{array}\right) \in \Gamma .
$$

Lemma 8.3. The matrix $\sigma_{1}^{ \pm}$transfers 0 -cells to 0 -cells as follows:

$$
\begin{aligned}
& \sigma_{1}^{+}\left[\theta_{1} \theta_{3} e_{13}, e_{16}, e_{25}\right]=\left[\theta_{1} \theta_{2} e_{14}, \theta_{2} e_{17}, \theta_{2} e_{26}\right] \\
& \sigma_{1}^{-}\left[e_{4}, e_{10}, \theta_{2} e_{12}, \theta_{2} e_{32}, \theta_{2} e_{33}, \theta_{2} e_{35}, \theta_{2} e_{37}\right] \\
& \quad=\left[e_{5}, e_{11}, \theta_{3} e_{15}, \theta_{3} \sigma_{0} e_{34}, \theta_{3} e_{34}, \theta_{3} e_{36}, \theta_{3} e_{38}\right] .
\end{aligned}
$$

Proof. This is easily proved by a direct computation.

Lemma 8.4. The sets $\mathcal{C}_{k}, \theta \mathcal{C}_{k}$ for $k=10,16,25,32,35,37$ are $\Gamma$-equivalent sets.

Proof. The equivalence of the sets $\mathcal{C}_{k}$ and $\theta \mathcal{C}_{k}$ in the assertion follows by Lemmas 8.1 and 8.3 with the help of $\sigma_{0}$ and the translations of $X_{i j}$-coordinates by \pm 1 .

Lemma 8.5. The sets $\mathcal{C}_{k}, \theta \mathcal{C}_{k}$ for $k=1,4,12,29$ are $\Gamma$ equivalent sets. 
Proof. The assertion when $k=1$ follows from the fact that

$$
\gamma_{1}=\left(\begin{array}{cc}
E_{2} & -I_{+} \\
E_{1} & E_{2}
\end{array}\right), \quad \gamma_{1} \cdot \theta_{3} e_{1}=\theta \theta_{3} e_{1} .
$$

Consider the case $k=4$. We can check that

$$
\gamma_{4}^{\prime}=\left(\begin{array}{cc}
E_{21}+I_{-} & -E_{2} \\
E_{2}-E_{12} & E_{1}
\end{array}\right), \quad \gamma_{4}^{\prime} \cdot e_{4}=e_{6} .
$$

By Lemma 8.3, we have $\sigma_{1}^{-} e_{4}=e_{5}$, so the equivalence of this case is now clear. When $k=12$, we need another equality:

$$
\gamma_{12}=\left(\begin{array}{cc}
E_{21} & E_{12} \\
-E_{12} & E_{21}
\end{array}\right), \quad \gamma_{12} \theta_{2} e_{12}=\theta \theta_{1} \sigma_{0} e_{13} .
$$

The equivalence when $k=12$ is now obtained by the help of Lemma 8.3.

To show the equivalence when $k=29$, it is enough to prove the following equality, which is obtained by direct computation:

$$
\gamma_{29}=\left(\begin{array}{cc}
I_{-}+E_{21} & -E_{2} \\
E_{2}-E_{12} & E_{1}
\end{array}\right), \quad \gamma_{29} e_{29}=\theta_{2} e_{30} .
$$

Lemma 8.6. The set $\mathcal{C}_{18}$ is a $\Gamma$-equivalent set.

Proof. Since $X_{i j}$ of $e_{18}$ are all $1 / 2$, the former eight elements in $\mathcal{C}_{18}$ are all $\Gamma$-equivalent. In particular, we have $e_{18} \stackrel{\Gamma}{\sim} \theta e_{18}$. Moreover, $\gamma_{18} e_{18}=\theta_{1} \theta_{3} e_{19}$ with $\gamma_{18}=$ $\left(\begin{array}{cc}E_{2} & E_{1} \\ -E_{1} & E_{2}\end{array}\right)$. Thus $e_{18} \stackrel{\Gamma}{\sim} \theta_{1} \theta_{3} e_{19}$. This means that

$$
\theta_{2} e_{19}=\theta \theta_{1} \theta_{3} e_{19}=\theta \gamma_{18} e_{18}=\gamma_{18}^{\theta} \theta e_{18} \stackrel{\Gamma}{\sim} e_{18} .
$$

Since $x_{11}=1 / 2$ for $e_{19}$, we have $e_{19} \stackrel{\Gamma}{\sim} \theta_{1} e_{19}$. Hence

$$
\theta_{1} \theta_{2} e_{19} \stackrel{\Gamma}{\sim} \theta_{2} e_{19} \stackrel{\Gamma}{\sim} e_{18}
$$

and $\theta_{3} e_{19} \stackrel{\Gamma}{\sim} \theta_{1} \theta_{3} e_{19} \stackrel{\Gamma}{\sim} e_{18}$.

We have to fix the cases $\theta_{3} e_{20}$ and $\theta_{1} \theta_{2} e_{20}$. Since $\theta_{2} e_{19} \stackrel{\Gamma}{\sim} e_{18}$, for $\theta_{3} e_{20}$ we apply the equality

$$
\gamma_{18}^{\prime} \theta_{2} e_{19}=\theta_{3} e_{20} \quad \text { for } \gamma_{18}^{\prime}=\left(\begin{array}{cc}
E_{1} & E_{2} \\
-E_{2} & E_{1}
\end{array}\right) .
$$

Finally,

$$
\begin{aligned}
\theta_{1} \theta_{2} e_{20} & =\theta\left(\theta_{3} e_{20}\right)=\theta \gamma_{18}^{\prime} \theta_{2} e_{19}=\left(\gamma_{18}^{\prime}\right)^{\theta} \theta \theta_{2} e_{19} \\
& =\left(\gamma_{18}^{\prime}\right)^{\theta} \theta_{1} \theta_{3} e_{19} \stackrel{\Gamma}{\sim} e_{18} .
\end{aligned}
$$

\begin{tabular}{l|ll} 
degree $/ \mathbb{Q}(\sqrt{-3})$ & $\mathbb{Q}(\mathcal{C})$ & $\mathcal{C}$ \\
\hline 1 & $\mathbb{Q}(\sqrt{-3})$ & $\mathcal{C}_{0}, \mathcal{C}_{3}$ \\
2 & $\mathbb{Q}(\sqrt{-3}, \sqrt{-1})$ & $\mathcal{C}_{7}, \theta \mathcal{C}_{7}$ \\
2 & $\mathbb{Q}\left(\omega_{4} \sqrt{-1}\right)$ & $\mathcal{C}_{9}$ \\
2 & $\mathbb{Q}\left(\omega_{5} \sqrt{-1}\right)$ & $\mathcal{C}_{10}$ \\
4 & $\mathbb{Q}\left(\omega_{6}+\sqrt{-1}\right)$ & $\mathcal{C}_{2}$ \\
4 & $\mathbb{Q}\left(\omega_{7}+\sqrt{-3}\right)$ & $\mathcal{C}_{16}, \theta \mathcal{C}_{16}$ \\
4 & $\mathbb{Q}\left(\omega_{21} \sqrt{-1}\right)$ & $\mathcal{C}_{18}$ \\
4 & $\mathbb{Q}\left(\nu_{15}\right)$ & $\mathcal{C}_{22}, \theta \mathcal{C}_{22}$ \\
8 & $\mathbb{Q}\left(\omega_{27} \sqrt{-1}\right)$ & $\mathcal{C}_{29}$ \\
8 & $\mathbb{Q}\left(\nu_{29}\right)$ & $\mathcal{C}_{32}, \theta \mathcal{C}_{32}$ \\
16 & $\mathbb{Q}\left(\nu_{31}\right)$ & $\mathcal{C}_{35}, \theta \mathcal{C}_{35}$ \\
18 & $\mathbb{Q}\left(\nu_{33}\right)$ & $\mathcal{C}_{37}, \theta \mathcal{C}_{37}$
\end{tabular}

TABLE 8. The field $\mathbb{Q}(\mathcal{C})$ that contains $\sqrt{-3}$. Here $\nu_{k}$ is such that $\left|\nu_{k}\right|=1$ and $\operatorname{Re}\left(\nu_{k}\right)=\omega_{k}$.

Summing up, we obtain the following theorem.

Theorem 8.7. Let $\mathcal{C}_{k}$ be the set defined as in Table 7 and let $\theta \mathcal{C}_{k}$ be its $\theta$-image. Then every $\mathcal{C}_{k}$ and every $\theta \mathcal{C}_{k}$ is a $\Gamma$-equivalence set.

Corollary 8.8. The upper bound of the number of $\Gamma$ equivalence classes $W_{0} / \stackrel{\Gamma}{\sim}$ is 40 .

\subsection{Inequivalence Property of $\mathcal{C}_{\boldsymbol{k}}$}

For $Z \in \mathbb{H}_{2}$, let $\mathbb{Q}(Z)$ denote $\mathbb{Q}\left(Z_{11}, Z_{12}, Z_{22}\right)$, the field generated by the point $Z$.

The following lemma is clear.

Lemma 8.9. If $\gamma Z=Z^{\prime}$ for $a \gamma \in \Gamma$, then $\mathbb{Q}(Z)=\mathbb{Q}\left(Z^{\prime}\right)$.

By Lemma $8.9, \mathbb{Q}(\mathcal{C})$ for the equivalence set $\mathcal{C}$ is well defined. We list these fields in Tables 8 and 9.

Proposition 8.10. The lower bound of the number of $\Gamma$ equivalence classes $W_{0} / \underset{\sim}{\sim}$ is 25 .

Proof. Since one can directly check that the fields listed in Tables 8 and 9 are all distinct, the possible number of $\Gamma$-equivalence classes is greater than or equal to 24 by Lemma 8.9.

To show that the lower bound is 25 , we prove that $\mathcal{C}_{0}$ and $\mathcal{C}_{3}$ are inequivalent by contradiction. Suppose there is an invertible integral matrix in $\Gamma$ that transfers $e_{0}$ to 


\begin{tabular}{l|ll} 
degree $/ \mathbb{Q}$ & $\mathbb{Q}(\mathcal{C})$ & $\mathcal{C}$ \\
\hline 2 & $\mathbb{Q}(\sqrt{-1})$ & $\mathcal{C}_{4}$ \\
2 & $\mathbb{Q}(\sqrt{-2})$ & $\mathcal{C}_{1}$ \\
4 & $\mathbb{Q}\left(\omega_{3} \sqrt{-1}\right)$ & $\mathcal{C}_{8}$ \\
6 & $\mathbb{Q}\left(\nu_{1}\right)$ & $\mathcal{C}_{12}, \theta \mathcal{C}_{12}$ \\
8 & $\mathbb{Q}\left(\nu_{14}\right)$ & $\mathcal{C}_{21}, \theta \mathcal{C}_{21}$ \\
8 & $\mathbb{Q}\left(\nu_{17}\right)$ & $\mathcal{C}_{23}, \theta \mathcal{C}_{23}$ \\
10 & $\mathbb{Q}\left(\nu_{20}\right)$ & $\mathcal{C}_{24}, \theta \mathcal{C}_{24}$ \\
12 & $\mathbb{Q}\left(\omega_{19} \sqrt{-1}\right) \mathcal{C}_{25}, \theta \mathcal{C}_{25}$ \\
12 & $\mathbb{Q}\left(\nu_{22}\right)$ & $\mathcal{C}_{27}, \theta \mathcal{C}_{27}$ \\
16 & $\mathbb{Q}\left(\nu_{28}\right)$ & $\mathcal{C}_{31}, \theta \mathcal{C}_{31}$ \\
24 & $\mathbb{Q}\left(\nu_{25}\right)$ & $\mathcal{C}_{28}, \theta \mathcal{C}_{28}$ \\
36 & $\mathbb{Q}\left(\nu_{36}\right)$ & $\mathcal{C}_{39}, \theta \mathcal{C}_{39}$
\end{tabular}

TABLE 9. The field $\mathbb{Q}(\mathcal{C})$ that does not contain $\sqrt{-3}$.

$e_{3}$. Then

$$
\left(A e_{0}+B\right)\left(C e_{0}+D\right)^{-1}=e_{3}
$$

and

$$
\eta A+B=\left(\begin{array}{cc}
\eta-1 & \eta / 2 \\
\eta / 2 & \eta-1
\end{array}\right) C+\left(\begin{array}{cc}
\eta & 1 / 2 \\
1 / 2 & \eta
\end{array}\right) D
$$

for $\eta=e^{\pi i / 3}$ and integral $2 \times 2$ matrices $A, B, C$, and $D$. By extraction, we readily find that both matrices $C$ and $D$ must have all entries even integers, a contradiction.

\subsection{Automorphic Functions and the Inequivalence Property: A Conjecture}

We conjecture that the all $\mathcal{C}_{j}$ are inequivalent, which should mean that $\left|W_{0} / \underset{\sim}{\sim}\right|=40$. To show this inequivalence property between $C_{j}$ 's, we should consider the case $k=7,12,26,21,22,23,24,25,27,28,31,32,35,37,39$. They are actually inequivalences between $\mathcal{C}$ and its $\theta$ image. In this case, however, $\mathbb{Q}(\mathcal{C})$ coincides with $\mathbb{Q}(\theta \mathcal{C})$, so we need additional information. Here we indicate a possible method to solve this question.

Let $J_{1}, J_{2}, J_{3}$ be the Igusa generators of the modular function fields $\mathbb{C}\left(J_{1}, J_{2}, J_{3}\right)$ of the Siegel modular variety $\Gamma \backslash \mathrm{H}_{2}$, which are the quotients of modular forms with adequate weights such that all the Fourier coefficients belong to $\mathbb{Q}$ [Igusa 62]. Then if $e_{j}$ and $\theta e_{j}$ are $\Gamma$-equivalent, one has

$$
J\left(e_{j}\right)=J\left(\theta e_{j}\right)=\overline{J\left(e_{j}\right)}, \quad J=J_{1}, J_{2}, J_{3} .
$$

This means that $e_{j} \in \Gamma \backslash \mathrm{H}_{2}$ defines a real point on the canonical model of $\mathbb{Q}\left(J_{1}, J_{2}, J_{3}\right)$. Therefore, our conjec- ture is equivalent to the claim that for each $j$, there exists $i$ such that $J_{i}\left(e_{j}\right) \notin \mathbb{R}$. We can check this condition if we can compute the numerical value of $J_{i}\left(e_{j}\right)$ with high-enough precision, applying the method of verified numerical computation [Rump et al. 08] if necessary.

\section{REFERENCES}

[Fricke and Klein 65] Robert Fricke and Felix Klein. Vorlesungen über die Theorie der automorphen Funktionen. Band 1: Die gruppentheoretischen Grundlagen. Band II: Die funktionentheoretischen Ausführungen und die Andwendungen, Bibliotheca Mathematica Teubneriana 4. New York: Johnson Reprint Corp., 1965.

[Gottschling 59] Erhard Gottschling. "Explizite Bestimmung der Randflächen des Fundamentalbereiches der Modulgruppe zweiten Grades." Math. Ann. 138 (1959), 103124.

[Gottschling 61a] Erhard Gottschling. "Über die Fixpunkte der Siegelschen Modulgruppe." Math. Ann. 143 (1961), 111-149.

[Gottschling 61b] Erhard Gottschling. "Über die Fixpunktuntergruppen der Siegelschen Modulgruppe." Math. Ann. 143 (1961), 399-430.

[Hashimoto 83] Ki-ichiro Hashimoto. "The Dimension of the Spaces of Cusp Forms on Siegel Upper Half-Plane of Degree Two. I." J. Fac. Sci. Univ. Tokyo Sect. IA Math. 30:2 (1983), 403-488.

[Igusa 62] Jun-ichi Igusa. "On Siegel Modular Forms of Genus Two." Amer. J. Math. 84 (1962), 175-200.

[Kamino 03] Itsuki Kamino. "On Fundamental Domains of Siegel Modular Groups of the Siegel Upper Half Plane of Degree 3" (Japanese). Master thesis, The University of Tokyo (H15)[2] No. 8010367921 (2003), 1-57.

[Kawamura 09] Takashi Kawamura. "The Minimum of the Determinant in the Fundamental Region of the Modular Group of Degree 2." Preprint, 2009.

[Klingen 90] Helmut Klingen. Introductory Lectures on Siegel Modular Forms, Cambridge Studies in Advanced Mathematics 20. Cambridge University Press, 1990.

[MacPherson and McConnell 93] Robert MacPherson and Mark McConnell. "Explicit Reduction Theory for Siegel Modular Threefolds." Invent. Math., 111:3 (1993), 575625 .

[Martinet 03] Jacques Martinet. Perfect Lattices in Euclidean Spaces, Grundlehren der Mathematischen Wissenschaften [Fundamental Principles of Mathematical Sciences] 327. Berlin: Springer-Verlag, 2003. 
[Noro and Yokoyama 03] Masayuki Noro and Kazuhiro Yokoyama. Computational Fundamentals of Gröbner Bases (Japanese). University of Tokyo Press, 2003.

[Rump et al. 08] Siegfried Rump, Takeshi Ogita, and Shin'ichi Oishi. "Accurate Floating-Point Summation. I. Faithful Rounding." SIAM J. Sci. Comput. 31:1 (2008), 189-224.

[Saito et al. 03] Tomokatsu Saito, Taku Takeshima, and Teluhiko Hilano. Practices on Gröbner Bases by Risa/Asir (Japanese). University of Tokyo Press, 2003.

[Serre 73] J.-P. Serre. A Course in Arithmetic. SpringerVerlag, 1973.

[Siegel 64] Carl Ludwig Siegel. Symplectic Geometry. Academic Press, 1964.

[Ueno 71] Kenji Ueno. "On Fibre Spaces of Normally Polarized Abelian Varieties of Dimension 2. I. Singular Fibres of the First Kind." J. Fac. Sci. Univ. Tokyo Sect. IA Math. 18 (1971), 37-95.

[Ueno 72] Kenji Ueno. On Fibre Spaces of Normally Polarized Abelian Varieties of Dimension 2. II. Singular Fibres of the First Kind. J. Fac. Sci. Univ. Tokyo Sect. IA Math. 19 (1972), 163-199.

[Watanabe 03] Takao Watanabe. "Fundamental Hermite Constants of Linear Algebraic Groups." J. Math. Soc. Japan 55:4 (2003), 1061-1080.

[Watanabe 11] Takao Watanabe. "A Survey on Voronoï's Theorem." in Geometry and Analysis of Automorphic Forms of Several Variables, edited by Yoshinori Hamahata et al., Series on Number Theory and Its Applications 7, pp. 334-377. Singapore: World Scientific, 2011.

[Yasaki 06] Dan Yasaki. "On the Existence of Spines for $\mathbb{Q}$ Rank 1 Groups." Selecta Math. (N.S.) 12:3-4 (2006), 541-564.

Takahiro Hayata, Department of Informatics, Faculty of Engineering, Yamagata University, 4-3-16, Jyonan, Yonezawashi, 992-8510, Japan (hayata@yz.yamagata-u.ac.jp)

Takayuki Oda, Graduate School of Mathematical Sciences, the University of Tokyo, 3-8-1, Komaba, Meguroku, Tokyo, 153-8914, Japan (takayuki@ms.u-tokyo.ac.jp)

Tomoki Yatougo, Department of Informatics, Faculty of Engineering, Yamagata University, 4-3-16, Jyonan, Yonezawashi, 992-8510, Japan (taw08385@st.yamagata-u.ac.jp) 